\title{
A Generalization of Nash Bargaining and Proportional Fairness to Log-Convex Utility Sets With Power Constraints
}

\author{
Holger Boche, Senior Member, IEEE, and Martin Schubert, Member, IEEE
}

\begin{abstract}
Many solutions and concepts in resource allocation and game theory rely on the assumption of a convex utility set. In this paper, we show that the less restrictive assumption of a logarithmic "hidden" convexity is sometimes sufficient. We consider the problems of Nash bargaining and proportional fairness, which are closely related. We extend the Nash bargaining framework to a broader family of log-convex sets. We then focus on the set of feasible signal-to-interference-plus-noise ratios (SINRs), for the cases of individual power constraints and a sum power constraint. Under the assumption of log-convex interference functions, we show how Pareto optimality of boundary points depends on the interference coupling between the users. Finally, we provide necessary and sufficient conditions for strict log-convexity of the feasible SINR region.
\end{abstract}

Index Terms-Game theory, interference, multiuser channels, Nash bargaining, power control, proportional fairness.

\section{INTRODUCTION}

$\mathbf{P}$ ERFORMANCE tradeoffs in multiuser systems occur when users share a common resource or if they are coupled by mutual interference. This is typical for wireless systems, and also for certain wireline connections, e.g., twisted-pair copper wires used for DSL transmission. The achievable performance is commonly characterized by the utility set, sometimes referred to as utility region or quality-of-service $(\mathrm{QoS})$ region. The utility region $\mathcal{U}$ is defined as the set of all achievable utility vectors $\boldsymbol{u}=\left[u_{1}, \ldots, u_{K}\right]^{T}$, where $K \geq 2$ is the number of users.

Many resource allocation strategies crucially depend on the structure of the set $\mathcal{U}$, so a thorough understanding of its boundary is needed. Some often-made assumptions are comprehensiveness, convexity, and Pareto optimality. Comprehensiveness can be interpreted as free disposability of utility. Convexity allows the application of well-known concepts from optimization and game theory. Pareto optimality is a notion

Manuscript received September 15, 2008; revised April 30, 2009; accepted April 22, 2010. Date of current version May 25, 2011. This work was supported in part by the Bundesministerium für Bildung und Forschung (BMBF) under Grant 01SF0708 (project TEROPP). The material in this paper was presented at the IEEE International Conference on Acoustics, Speech, and Signal Processing, Taipei, Taiwan, R.O.C., April 2009.

$\mathrm{H}$. Boche was with the Fraunhofer Institute for Telecommunications and the Technical University of Berlin, Germany. He is now with the Technische Universität München, Germany.

M. Schubert is with the Fraunhofer Institute for Telecommunications HHI, Berlin, Germany.

Communicated by A. van Wijngaarden, Associate Editor for Communications.

Digital Object Identifier 10.1109/TIT.2011.2132470 of efficiency, requiring that one cannot make someone better off without making someone else worse off. A Pareto optimal operating point is "efficient" in the sense that the available system resources are fully utilized.

Whether these properties are fulfilled or not depends on many factors, including the choice of the performance measure, and system constraints. If the users are coupled by mutual interference then interference mitigation techniques play an important role. This can lead to complicated "cross-layer" dependencies with intractable problem formulations. In order to analyze the utility tradeoffs between the users, we need to make some assumptions regarding the underlying system. In this paper, the following cases will be studied.

Strictly log-convex utility sets (Section II). This class is broader than the class of convex sets, which is contained as a special case. It is shown that the core properties of the game-theoretic framework of Nash bargaining [1] are preserved under strict log-convexity. In this case, the Nash bargaining solution is equivalent to proportional fairness [2].

Log-convex interference functions (Sections III and IV). The utilities are assumed to be strictly monotone and continuous functions of the signal-to-interference-plus-noise ratios (SINRs). The interference coupling between the users is modeled by means of axiomatic log-convex interference functions [3]. Power constraints are assumed. Based on the system dependency matrix, we derive a necessary and sufficient condition for Pareto optimality of boundary points.

In Section V we provide a sufficient condition for an SINR set from Section III to be strictly log-convex. If this is fulfilled, then we known from the results of Section II that a unique Nash bargaining solution exists. These results extend previous work [3], where only log-convexity was shown and [4] where strict log-convexity was only shown for linear interference functions.

These results have in common that they all rely on a logarithmic convexity. In Section II the utility set itself is assumed to be log-convex, while in Sections III-V we assume log-convex interference functions.

Note, that the requirement of log-convexity is weaker than the customary assumption of convexity. Thus the results are applicable to a broader class of problems. Log-convexity is a useful property that allows the application of convex optimization techniques to certain nonconvex problems. This is sometimes referred to as "hidden convexity." Log-convexity was already successfully exploited in different other contexts, e.g., [5]-[11].

An important aspect of our work is the presence of power constraints. Without power constraints, strict log-convexity follows 
easily from the results [3] if the underlying interference functions are strictly log-convex. However, with power constraints, and especially individual power constraints, proving strictness requires a different mathematical approach involving combinatorial arguments.

We use the following notational conventions. Matrices and vectors are denoted by bold capital letters and bold lowercase letters, respectively. The $k$ th component of a vector $\boldsymbol{y}$ is denoted by $y_{l}$ or, equivalently, by $[\boldsymbol{y}]_{l}$. The notation $\boldsymbol{y} \geq 0$ means that $y_{l} \geq 0$ for all components $l$, and $\boldsymbol{x} \supsetneqq \boldsymbol{y}$ means componentwise inequality with strict inequality for at least one component. Similar definitions hold for the reverse directions. Finally, $\boldsymbol{x} \neq \boldsymbol{y}$ means that the vectors differ in at least one component. The set of nonnegative reals is denoted as $\mathbb{R}_{+}$, and the set of positive reals is denoted as $\mathbb{R}_{++}$.

\section{EXTENSION OF NASH BARGAINING TO LOG-CONVEX UTILITY SETS}

Strategies for distributing the system resources are usually based on certain notions of "fairness" or "efficiency." In this section, we focus on the game-theoretic strategy of Nash bargaining [1], [12], [13], which is closely related to proportional fairness [2]. This approach is cooperative, i.e., users (or players) unanimously agree on some solution outcome $\varphi(\mathcal{U})$. This outcome is generally better than the Nash equilibrium resulting from a noncooperative approach. The gain from cooperation can be substantial (see, e.g., [14] and [15]). Nash bargaining was successfully applied to various multiuser communication scenarios, e.g., [16]-[21].

In this section, we will show that the conventional Nash bargaining framework can be extended to certain log-convex utility sets.

Our approach is based on a change of variable $q_{k}=\log u_{k}$, where $u_{k}$ is the utility of the $k$ th user. This is a common technique for exploiting hidden convexity (see, e.g., [5]-[11]).

\section{A. The Conventional Nash Bargaining Solution (NBS)}

We begin by briefly reviewing the NBS, which was introduced by Nash [1] and extended later (see, e.g., [12], [13], [22] and references therein). The NBS in its standard form requires that the utility set $\mathcal{U}$ is convex. In this section we will generalize these results to certain nonconvex sets.

Definition 1: A bargaining game for $K$ users is defined as a pair $(\mathcal{U}, \boldsymbol{d})$, where

- $\mathcal{U}$ is a nonempty compact subset of $\mathbb{R}_{+}^{K}$.

- $\mathcal{U}$ is (downward)-comprehensive. That is, for all $\boldsymbol{u} \in \mathcal{U}$ and $\boldsymbol{u}^{\prime} \in \mathbb{R}_{+}^{K}$, the component-wise inequality $\boldsymbol{u}^{\prime} \leq \boldsymbol{u}$ implies $\boldsymbol{u}^{\prime} \in \mathcal{U}$.

- $\boldsymbol{d} \in\left\{\boldsymbol{u} \in \mathcal{U}: \exists \boldsymbol{u}^{\prime}>\boldsymbol{u}\right\}$ is the disagreement point, which is the outcome in case that no agreement can be found.

The class of sets with these properties is denoted by $\mathcal{D}^{K}$.

Definition 2: Let $\mathcal{U} \in \mathcal{D}^{K}$ be convex, then the NBS is the unique (single-valued) solution that fulfills the following axioms.
- Weak Pareto Optimality (WPO). The users should not be able to collectively improve upon the solution outcome, i.e.,

$\varphi(\mathcal{U}) \in\left\{\boldsymbol{u} \in \mathcal{U}:\right.$ there is no $\boldsymbol{u}^{\prime} \in \mathcal{U}$ with $\left.\boldsymbol{u}^{\prime}>\boldsymbol{u}\right\}$

- Symmetry (SYM). If $\mathcal{U}$ is symmetric, ${ }^{1}$ then the outcome does only depend on the employed strategies and not on the identities of the users, i.e., $\varphi_{1}(\mathcal{U})=\cdots=\varphi_{K}(\mathcal{U})$. This does not mean that the utility set is symmetric, but rather that all users have the same priorities.

- Independence of Irrelevant Alternatives (IIA). If $\varphi(\mathcal{U})$ is the solution outcome of some utility set $\mathcal{U}$, then $\varphi(\mathcal{U})$ is also the solution outcome of every subset containing $\varphi(\mathcal{U})$, i.e.,

$$
\varphi(\mathcal{U}) \in \mathcal{U}^{\prime}, \text { with } \mathcal{U}^{\prime} \subseteq \mathcal{U} \Longrightarrow \varphi\left(\mathcal{U}^{\prime}\right)=\varphi(\mathcal{U})
$$

- Scale Transformation Covariance (STC). The optimization strategy is invariant with respect to a component-wise scaling of the region. ${ }^{2}$ That is, for every $\mathcal{U} \in \mathcal{D}^{K}$, and all $\boldsymbol{a}, \boldsymbol{b} \in \mathbb{R}^{K}$ with $\boldsymbol{a}>0$ and $(\boldsymbol{a} \circ \mathcal{U}+\boldsymbol{b}) \in \mathcal{D}^{K}$, we have

$$
\varphi(\boldsymbol{a} \circ \mathcal{U}+\boldsymbol{b})=\boldsymbol{a} \circ \varphi(\mathcal{U})+\boldsymbol{b} .
$$

If the utility set $\mathcal{U}$ is compact convex comprehensive, then the single-valued NBS fulfilling the four axioms is obtained by maximizing the product of utilities (Nash product).

$$
\max _{\boldsymbol{u} \in \mathcal{U}, \boldsymbol{u} \geq \boldsymbol{d}} \prod_{k \in \mathcal{K}}\left(u_{k}-d_{k}\right) .
$$

In this paper, $\boldsymbol{d}=\mathbf{0}$. This assumption is often made if the choice of the zero of the utility scales does not matter. This also makes sense in a wireless system which is subject to channel fluctuations, so no minimum performance can be guaranteed. This leads to the problem formulation

$$
\max _{\boldsymbol{u} \in \mathcal{U}} \prod_{k \in \mathcal{K}} u_{k}
$$

Since $\log \max \prod_{k} u_{k}=\max \log \prod_{k} u_{k}=\max \sum_{k} \log u_{k}$, the optimum (2) can be found by solving

$$
\max _{\boldsymbol{u} \in \mathcal{U}} \sum_{k \in \mathcal{K}} \log u_{k}
$$

In the following, we will refer to strategy (3) as proportional fairness (PF). In its original definition [2], a vector $\boldsymbol{u}^{*}$ is said to be proportionally fair if for any other feasible vector $\boldsymbol{u} \in \mathcal{U}$ the aggregated proportional change $\sum_{k}\left(u_{k}-u_{k}^{*}\right) / u_{k}^{*}$ is nonpositive (see also [23]). For convex sets, this unique point is obtained as the optimizer of (3). In this case, Nash bargaining and proportional fairness are equivalent [2], [16]. This relates the NBS to

\footnotetext{
${ }^{1} \mathrm{~A}$ game $(\mathcal{U}, \boldsymbol{d})$ is said to be symmetric if $d_{1}=\cdots=d_{K}$, and in addition, $\boldsymbol{u}=\left[u_{1}, \ldots, u_{K}\right] \in \mathcal{U} \Leftrightarrow \boldsymbol{u}^{\prime}=\left[u_{\pi_{1}}, \ldots, u_{\pi_{K}}\right] \in \mathcal{U}$, for an arbitrary permutation $\pi$.

${ }^{2}$ We use the component-wise Hadamard product $\circ$, and the notation $\boldsymbol{a} \circ \mathcal{U}=$ $\{\boldsymbol{u}: \exists \boldsymbol{s} \in \mathcal{U}$ with $\boldsymbol{u}=\boldsymbol{a} \circ \boldsymbol{s}\}$.
} 
a known fairness criterion (see also [16], [17], [19], [20], and [24]).

In this paper we are interested in certain nonconvex sets which are strictly convex after a logarithmic transformation (strict log-convexity). Because of nonconvexity, it remains to be shown whether (3) is still equivalent to the original definition of proportional fairness [25]. We will refer to (3) as "proportional fairness." It will be shown that the property of strict log-convexity is sufficient to ensure a unique optimizer of (3).

\section{B. Logarithmic Transformation}

For every compact convex set from $\mathcal{D}^{K}$, the product maximizer (2) is the single-valued NBS characterized by the axioms WPO, SYM, IIA, and STC. However, convexity does not need to be fulfilled. An example is the SINR region discussed in Section III.

A standard approach is to convexify the utility set based on randomization arguments (see, e.g., [21] and [26]), or by resource sharing. However, such a strategy is not always possible or even relevant. Again, the SINR region provides an example of a performance measure for which convexification is difficult to justify. Extensions and modifications of the NBS to nonconvex utility sets have been studied in the literature, e.g., [26]-[29]. However, the motivation of these papers is quite different from our approach.

In this section we will show that the key properties of the standard Nash bargaining framework extend to certain nonconvex sets. To this end, consider the function $\log (\boldsymbol{u})=\left[\log u_{1}, \ldots, \log u_{K}\right]^{T}$, where $\boldsymbol{u} \in \mathcal{U} \cap \mathbb{R}_{++}^{K}$. The image set of $\mathcal{U}$ is

$$
\mathcal{L} \operatorname{og}(\mathcal{U}):=\left\{\boldsymbol{q}=\log (\boldsymbol{u}): \boldsymbol{u} \in \mathcal{U} \cap \mathbb{R}_{++}^{K}\right\} .
$$

Definition 3: We say that a set $\mathcal{U} \subseteq \mathbb{R}_{+}^{K}$ is a log-convex set if $\log (\mathcal{U})$ is convex.

The boundary of the utility set $\mathcal{U}$ is denoted by $\partial \mathcal{U}$.

Definition 4: A boundary point $\boldsymbol{u} \in \partial \mathcal{U}$ is said to be Pareto optimal if there is no $\hat{\boldsymbol{u}} \in \partial \mathcal{U}$ with $\hat{\boldsymbol{u}} \supsetneqq \boldsymbol{u}$. The set of all Pareto optimal boundary points (the Pareto boundary) is denoted by $P O(\mathcal{U})$.

From a practical point of view, Pareto optimality means that it is not possible to improve the performance of one user without decreasing the performance of another user.

Definition 5: By $\mathcal{S} \mathcal{T}$ we denote the family of all closed downward-comprehensive utility sets $\mathcal{U} \subset \mathbb{R}_{+}^{K}$ such that the image set $\mathcal{Q}:=\log (\mathcal{U})$ is convex and the following additional property is fulfilled: For any $\hat{\boldsymbol{q}}, \check{\boldsymbol{q}} \in P O(\mathcal{Q})$, the connecting line $\boldsymbol{q}(\lambda)=(1-\lambda) \hat{\boldsymbol{q}}+\lambda \check{\boldsymbol{q}}$, with $\lambda \in(0,1)$, is contained in the interior of $\mathcal{Q}$. By $\mathcal{S} \mathcal{T}_{c}$ we denote the family of all $\mathcal{U} \in \mathcal{S T}$, which are additionally bounded, thus compact.

Definition 5 is illustrated in Fig. 1.

Note that compactness and comprehensiveness are preserved by the log-transformation. That is, $\mathcal{U} \subset \mathbb{R}_{+}^{K}$ is compact comprehensive if and only if $\log (\mathcal{U}) \subset \mathbb{R}^{K}$ is compact comprehensive. Every convex set from $\mathcal{D}^{K}$ is contained in $\mathcal{S} \mathcal{T}_{c}$, but not conversely. Thus, $\mathcal{S T}_{c}$ is more general than the class of standard sets described by Definition 1. In the following we show

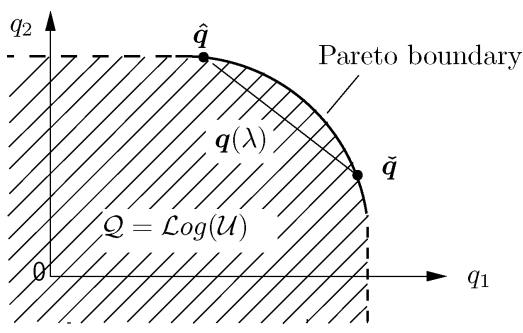

Fig. 1. Illustration of an image set $\mathcal{Q}:=\log (\mathcal{U})$ for $\mathcal{U} \in \mathcal{S} \mathcal{T}_{c}$. The set is strictly convex with the exception of possible boundary segments parallel to the axes (dashed lines). These segments are irrelevant for the Nash solution.

that for any $\mathcal{U} \in \mathcal{S} \mathcal{T}_{c}$, product maximization (2) yields the single-valued NBS characterized by axioms WPO, SYM, IIA, and STC. This extends the classical Nash bargaining framework to certain nonconvex sets.

The properties of $\mathcal{S} \mathcal{T}_{c}$ play an important role for the proof of uniqueness. We also exploit that the axioms WPO, SYM, IIA, and STC have direct counterparts for the image set $\mathcal{Q}:=$ $\log (\mathcal{U})$. This is straightforward for axioms WPO, SYM, and IIA, which are not affected by the logarithmic transformation. That is, axiom WPO in the utility set $\mathcal{U}$ corresponds directly to WPO in the image set $\mathcal{Q}$. The same holds for axioms SYM and IIA. We will denote the axioms associated with the image set by $\mathrm{WPO}_{\mathcal{Q}}, \mathrm{SYM}_{\mathcal{Q}}$, and $\mathrm{IIA}_{\mathcal{Q}}$.

Axiom STC in the utility set $\mathcal{U} \in \mathcal{S} \mathcal{T}_{c}$ also has a direct correspondence for the image set $\mathcal{Q}:=\operatorname{L} o g(\mathcal{U})$. Consider an arbitrary translation $\tilde{\boldsymbol{q}} \in \mathbb{R}^{K}$, leading to a translated set $\mathcal{Q}(\tilde{\boldsymbol{q}})$, defined as

$$
\mathcal{Q}(\tilde{\boldsymbol{q}})=\left\{\boldsymbol{q} \in \mathbb{R}^{K}: \exists \boldsymbol{q}_{0} \in \mathcal{Q} \text { with } \boldsymbol{q}=\boldsymbol{q}_{0}+\tilde{\boldsymbol{q}}\right\} .
$$

Also, let $\varphi_{\mathcal{Q}}$ be the log-transformed Nash bargaining solution, i.e., $\varphi_{\mathcal{Q}}=\log \varphi(\mathcal{U})$. Since the disagreement point is zero in our case, axiom STC becomes $\varphi(\boldsymbol{a} \circ \mathcal{U})=a \circ \varphi(\mathcal{U})$. In the $\log$-transformed domain, this corresponds to

$$
\varphi_{\mathcal{Q}}(\mathcal{Q}(\tilde{\boldsymbol{q}}))=\varphi_{\mathcal{Q}}(\mathcal{Q})+\tilde{\boldsymbol{q}} .
$$

We will refer to (5) as $\mathrm{STC}_{\mathcal{Q}}$.

It is now shown that the transformed axioms are associated with a unique solution outcome $\varphi_{\mathcal{Q}}$ in the transformed set.

Theorem 1: For an arbitrary set $\mathcal{U} \in \mathcal{S} \mathcal{T}_{c}$, the solution outcome $\varphi_{\mathcal{Q}}$ in the transformed set $\mathcal{Q}=\log (\mathcal{U})$ satisfies axioms $\mathrm{WPO}_{Q}, \mathrm{SYM}_{Q}, \mathrm{STC}_{Q}$, and IIA ${ }_{Q}$ if and only if it is the unique maximizer

$$
\boldsymbol{\varphi}_{\mathcal{Q}}(\mathcal{Q})=\underset{\boldsymbol{q} \in \mathcal{Q}}{\arg \max } \sum_{k \in \mathcal{K}} q_{k} .
$$

Proof: Non-Pareto-optimal boundary segments parallel to the axes can be safely excluded from the proof, since such points cannot be the solution of the product maximization (2). Thus, without loss of generality we can assume that $\mathcal{Q}$ is strictly convex.

Given the properties of the region $\mathcal{U} \in \mathcal{S} \mathcal{T}_{c}$ and its image set $\log (\mathcal{U})$, it is clear that the solution (6) satisfies the axioms $\mathrm{WPO}_{Q}, \mathrm{SYM}_{Q}, \mathrm{STC}_{Q}$, and $\mathrm{IIA}_{Q}$. 


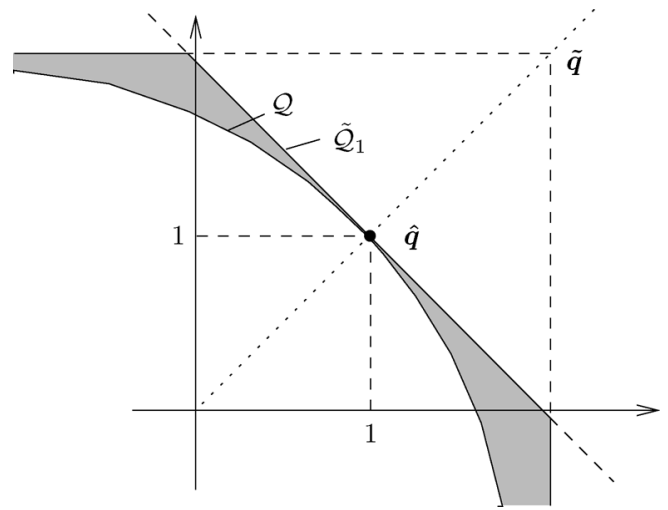

Fig. 2. Illustration of the proof of Theorem 1. The NBS in the transformed set $\mathcal{Q}$ is the unique solution that satisfies the transformed axioms.

It remains to show the converse. Consider a bargaining strategy on $\mathcal{Q}=\mathcal{L} \circ g(\mathcal{U})$, that satisfies the axioms $\mathrm{WPO}_{Q}$, $\mathrm{SYM}_{Q}, \mathrm{STC}_{Q}, \mathrm{IIA}_{Q}$. We now show that these axioms are fulfilled by a unique solution, which is the optimizer of (6). This is illustrated by Fig. 2.

Consider the set

$$
\mathcal{Q}_{1}:=\left\{\boldsymbol{q} \in \mathbb{R}^{K}: \sum_{k \in \mathcal{K}} q_{k} \leq K\right\}
$$

Because of the $\mathrm{STC}_{\mathcal{Q}}$ property (5), we know that the strategy is invariant with respect to a translation of the region. Thus, without loss of generality we can assume $\mathcal{Q} \subseteq \mathcal{Q}_{1}$, and

$$
\hat{\boldsymbol{q}}=[1, \ldots, 1]^{T}=\underset{\boldsymbol{q} \in \mathcal{Q}}{\arg \max } \sum_{k \in \mathcal{K}} q_{k} .
$$

That is, $\hat{\boldsymbol{q}}$ is the unique point which is on the boundaries of both sets $\mathcal{Q}$ and $\mathcal{Q}_{1}$. Since $\mathcal{Q}$ is upper-bounded by definition, there is a $\tilde{\boldsymbol{q}} \in \mathbb{R}^{K}$ such that

$$
\tilde{\boldsymbol{q}} \geq \boldsymbol{q} \text { for all } \boldsymbol{q} \in \mathcal{Q} \text {. }
$$

Thus, $\mathcal{Q}$ is a subset of the set

$$
\tilde{\mathcal{Q}}_{1}=\left\{\boldsymbol{q} \in \mathcal{Q}_{1}: \boldsymbol{q} \leq \tilde{\boldsymbol{q}}\right\} .
$$

The set $\tilde{\mathcal{Q}}_{1}$ is symmetric and strictly convex. Let $\tilde{\mathcal{Q}}$ be the smallest symmetric and strictly convex closed set that fulfills

$$
\tilde{\mathcal{Q}}_{1} \supseteq \tilde{\mathcal{Q}} \supseteq \mathcal{Q} .
$$

Since $\tilde{\mathcal{Q}}_{1}$ is upper-bounded, the set $\tilde{\mathcal{Q}}$ is compact. It is also strictly convex comprehensive, thus it is contained in $\log \left(\mathcal{S \mathcal { T } _ { c } )}\right.$, which is the class of all sets $\log (\mathcal{U})$ such that $\mathcal{U} \in \mathcal{S} \mathcal{T}_{c}$. Because of axiom $\mathrm{SYM}_{Q}$, it follows that

$$
\varphi_{\mathcal{Q}}(\tilde{\mathcal{Q}})=\hat{\boldsymbol{q}}=[1, \ldots, 1]^{T} .
$$

$\sum_{k} q_{k}=K$ describes a supporting hyperplane for $\tilde{\mathcal{Q}}$, i.e., $\hat{\boldsymbol{q}}$ is an optimizer of

$$
\boldsymbol{\varphi}_{\mathcal{Q}}(\tilde{\mathcal{Q}})=\underset{\boldsymbol{q} \in \tilde{\mathcal{Q}}}{\arg \max } \sum_{k \in \mathcal{K}} q_{k}
$$

Now, $\mathcal{Q} \subseteq \tilde{\mathcal{Q}}$ and $\hat{\boldsymbol{q}} \in \mathcal{Q}$. Because of axiom $\operatorname{IIA}_{\mathcal{Q}}$, we have

$$
\varphi_{\mathcal{Q}}(\mathcal{Q})=\varphi_{\mathcal{Q}}(\tilde{\mathcal{Q}})=[1, \ldots, 1]^{T}=\underset{\boldsymbol{q} \in \mathcal{Q}}{\arg \max } \sum_{k \in \mathcal{K}} q_{k}
$$

which concludes the proof.

Consequently, for all $\mathcal{U} \in \mathcal{S} \mathcal{T}_{c}$ the optimization (9) in the transformed domain $\mathcal{Q}=\log (\mathcal{U})$ leads to the unique optimum $\varphi_{\mathcal{Q}}(\mathcal{Q})$. Because of the one-to-one logarithmic mapping between the sets $\mathcal{Q}$ and $\mathcal{U}$, we have the following result.

Corollary 1: Let $\mathcal{U} \in \mathcal{S T}_{c}$. Then axioms WPO, SYM, STC, and IIA are satisfied by the unique solution

$$
\varphi(\mathcal{U})=\underset{\boldsymbol{u} \in \mathcal{U}}{\arg \max } \prod_{k \in \mathcal{K}} u_{k} .
$$

This result holds for arbitrary utility sets from $\mathcal{S T}$, including the conventional case of convex sets. In the remainder we will study a wireless communication scenario where such a nonconvex set from $\mathcal{S} \mathcal{T}_{c}$ occurs. This will be discussed later in Section V.

\section{INTERFERENCE-COUPLED WIRELESS SYSTEMS BASED ON LOG-CONVEX INTERFERENCE FUNCTIONS}

In this section we study the SINR region of an interference-coupled multiuser system with power constraints. It will be shown how Pareto optimality of boundaries points (see Definition 4) depends on the interference coupling between the users.

Consider $K$ users with transmit powers $\boldsymbol{p}=\left[p_{1}, \ldots, p_{K}\right]^{T}$. The noise power at each receiver is $\sigma_{n}^{2}$. Hence, the SINR at each receiver depends on the extended power vector

$$
\underline{\boldsymbol{p}}=\left[\begin{array}{c}
\boldsymbol{p} \\
\sigma_{n}^{2}
\end{array}\right]=\left[p_{1}, \ldots, p_{K}, \sigma_{n}^{2}\right]^{T} .
$$

The resulting SINR of user $k$ is $\operatorname{SINR}_{k}(\underline{\boldsymbol{p}})=p_{k} / \mathcal{I}_{k}(\underline{\boldsymbol{p}})$, where $\mathcal{I}_{k}$ is the interference (plus noise) as a function of $\underline{\boldsymbol{p}}$.

\section{A. Interference Functions}

An axiomatic approach to interference modeling was proposed by Yates in [30], and extended in [31], [32]. In this work, mutual interference was modeled by a framework of axioms

Definition 6: A function $Y: \mathbb{R}_{+}^{K} \mapsto \mathbb{R}_{++}$is said to be a standard interference function if the following axioms are fulfilled: Y1 (positivity): $Y(\boldsymbol{p})>0$ for all $\boldsymbol{p} \in \mathbb{R}_{+}^{K}$, Y2 (scalability): $\alpha Y(\boldsymbol{p})>Y(\alpha \boldsymbol{p})$ for all $\alpha>1$, Y3 (monotonicity): $Y(\boldsymbol{p}) \geq Y\left(\boldsymbol{p}^{\prime}\right)$ if $\boldsymbol{p} \geq \boldsymbol{p}^{\prime}$.

This model is sufficiently general to incorporate cross-layer effects and it serves as a theoretical basis for many algorithms. Examples include beamforming [33]-[36], CDMA [37], [38], base station assignment [39], [40], and robust designs [41], [42].

In this paper we use a different axiomatic framework that was introduced in [43].

Definition 7: The function $\mathcal{I}: \mathbb{R}_{+}^{K+1} \mapsto \mathbb{R}_{+}$is an interference function if the following axioms are fulfilled:

A1 (positivity): There is a $\underline{p}>0$ with $\mathcal{I}(\underline{p})>0$,

A2 (scale invariance): $\mathcal{I}(\alpha \underline{\boldsymbol{p}})=\alpha \mathcal{I}(\underline{\boldsymbol{p}})$ for all $\alpha>0$, 
A3 (monotonicity): $\mathcal{I}(\boldsymbol{p}) \geq \mathcal{I}\left(\boldsymbol{p}^{\prime}\right)$ if $\boldsymbol{p} \geq \boldsymbol{p}^{\prime}$.

In addition, we require strict monotonicity with respect to the noise component.

A4 (strict monotonicity): $\mathcal{I}(\underline{\boldsymbol{p}})>\mathcal{I}\left(\underline{\boldsymbol{p}}^{\prime}\right)$ if $\underline{\boldsymbol{p}} \geq \underline{\boldsymbol{p}}^{\prime}$ and $\underline{p}_{K+1}>\underline{p}_{K+1}^{\prime}$.

A simple example is

$$
\mathcal{I}(\underline{\boldsymbol{p}})=\boldsymbol{v}^{T} \boldsymbol{p}+\sigma_{n}^{2}
$$

where $\boldsymbol{v} \in \mathbb{R}_{+}^{K}$ is a vector of interference coupling coefficients.

The axiomatic framework A1-A4 is closely connected with the standard interference functions. For any constant noise power $\underline{p}_{K+1}=\sigma_{n}^{2}$, the function $Y_{\mathcal{I}}(\boldsymbol{p})=\mathcal{I}(\underline{\boldsymbol{p}})$ is standard. Conversely, any standard interference function can be expressed within the framework A1-A4 [44]. Let $Y$ be a standard interference function, then

$$
\mathcal{I}_{Y}(\underline{\boldsymbol{p}})=\underline{p}_{K+1} \cdot Y\left(\frac{p_{1}}{\underline{p}_{K+1}}, \ldots, \frac{p_{K}}{\underline{p}_{K+1}}\right)
$$

is an interference function fulfilling A1-A4.

For more details about the relationship between the framework A1-A4 and Yates' standard interference functions, the reader is referred to [43]. For the purpose of this paper it is sufficient to know that there is such a connection and that all the results of this paper are also applicable to standard interference functions. The reason for using $\mathrm{A} 1-\mathrm{A} 4$ is that it enables us to use previous results [3], where logarithmic convexity was studied. Some of the following results use this property.

\section{B. The SINR Region Under Power Constraints}

Consider the feasible SINR region for users $\mathcal{K}=$ $\{1,2, \ldots, K\}$, with individual power constraints $\boldsymbol{p} \leq \boldsymbol{p}^{\max }=$ $\left[p_{1}^{\max }, \ldots, p_{K}^{\max }\right]^{T}$, which is defined as the sublevel set

$$
\mathcal{S}\left(\mathcal{I}, \boldsymbol{p}^{\max }\right)=\left\{\boldsymbol{\gamma} \in \mathbb{R}_{++}^{K}: C\left(\boldsymbol{\gamma}, \mathcal{I}, \boldsymbol{p}^{\max }\right) \leq 1\right\}
$$

where $\mathcal{I}=\left[\mathcal{I}_{1}, \ldots, \mathcal{I}_{K}\right]^{T}$ and $\gamma$ is a vector of SINR values.

A point $\boldsymbol{\gamma} \in \mathbb{R}_{+}^{K}$ is feasible if $C\left(\boldsymbol{\gamma}, \mathcal{I}, \boldsymbol{p}^{\max }\right) \leq 1$, where

$$
C\left(\boldsymbol{\gamma}, \mathcal{I}, \boldsymbol{p}^{\max }\right)=\inf _{\boldsymbol{0}<\boldsymbol{p} \leq \boldsymbol{p}^{\max }}\left(\max _{k \in \mathcal{K}} \frac{\gamma_{k} \mathcal{I}_{k}(\underline{\boldsymbol{p}})}{p_{k}}\right) .
$$

The structure of the SINR set $\mathcal{S}\left(\mathcal{I}, \boldsymbol{p}^{\max }\right)$ depends on the properties of the indicator function $C\left(\boldsymbol{\gamma}, \mathcal{I}, \boldsymbol{p}^{\max }\right)$, which in turn depends on the properties of the underlying interference functions $\mathcal{I}_{1}, \ldots, \mathcal{I}_{K}$, as well as on the chosen power constraints $\boldsymbol{p}^{\max }$.

It can be observed that $C\left(\boldsymbol{\gamma}, \mathcal{I}, \boldsymbol{p}^{\max }\right)$ itself is an "interference function" fulfilling the core axioms A1, A2, A3. This was exploited in [45], where it was shown that any comprehensive subset of $\mathbb{R}_{+}^{K}$ can be expressed as a sublevel set of an interference function. Sublevel sets of convex functions are convex, thus $\mathcal{S}\left(\mathcal{I}, \boldsymbol{p}^{\max }\right)$ is a closed convex set from $\mathbb{R}_{+}^{K}$ if $C\left(\boldsymbol{\gamma}, \mathcal{I}, \boldsymbol{p}^{\max }\right)$ is convex. However, convexity of $C\left(\boldsymbol{\gamma}, \mathcal{I}, \boldsymbol{p}^{\max }\right)$ does generally not hold, so SINR regions are typically nonconvex.

The SINR region under a sum power constraint is defined as

$$
\mathcal{S}\left(\mathcal{I}, P_{\text {tot }}\right)=\left\{\boldsymbol{\gamma} \in \mathbb{R}_{++}^{K}: C\left(\gamma, \mathcal{I}, P_{\text {tot }}\right) \leq 1\right\}
$$

where

$$
C\left(\boldsymbol{\gamma}, \mathcal{I}, P_{t o t}\right)=\inf _{\boldsymbol{p}>0 ;\|\boldsymbol{p}\|_{1} \leq P_{t o t}}\left(\max _{k \in \mathcal{K}} \frac{\gamma_{k} \mathcal{I}_{k}(\underline{\boldsymbol{p}})}{p_{k}}\right) .
$$

Both regions $\mathcal{S}\left(\mathcal{I}, \boldsymbol{p}^{\max }\right)$ and $\mathcal{S}\left(\mathcal{I}, P_{\text {tot }}\right)$ will be analyzed in the following, where it will turn out that the sum-power constrained region $\mathcal{S}\left(\mathcal{I}, P_{\text {tot }}\right)$ is relatively easy to handle because the users are connected via a shared power budget. The region $\mathcal{S}\left(\mathcal{I}, \boldsymbol{p}^{\max }\right)$ is more complicated in that tradeoffs generally depend on interference coupling. Therefore, a large part of our analysis will focus on the effects of interference coupling.

In this paper we focus on log-convex interference functions, which will be introduced in the following section. Log-convex interference functions are rich in terms of analytical possibilities. This will be exploited in the remainder of this paper, where we will study conditions for Pareto optimality and strict logarithmic convexity of the SINR regions.

\section{Log-Convex Interference Functions}

We will now discuss the important subclass of log-convex interference functions. Throughout this paper, all interference functions are assumed to be log-convex.

For an explanation, consider the function $f(\underline{s})$ := $\mathcal{I}(\exp \{\underline{s}\})$, which is said to be $\log$-convex on $\mathbb{R}^{K+1}$ if $\log f$ is convex, or equivalently [46]

$$
f((1-\lambda) \underline{\hat{\boldsymbol{s}}}+\lambda \underline{\check{\boldsymbol{s}}}) \leq f(\underline{\hat{\boldsymbol{s}}})^{1-\lambda} f(\underline{\underline{s}})^{\lambda} \quad \forall \lambda \in(0,1)
$$

for arbitrary $\underline{\hat{s}}, \underline{s} \in \mathbb{R}^{K+1}$.

Definition 8: The interference function $\mathcal{I}$ is a log-convex interference function if $\mathcal{I}(\exp \{\underline{\boldsymbol{s}}\})$ is log-convex on $\mathbb{R}^{K+1}$.

Note that the log-convexity in Definition 8 is based on a change of variable $\boldsymbol{p}=\exp \{\boldsymbol{s}\}$ (component-wise exponential). Such a technique was already used by Sung [5] in the context of linear interference functions, and later in [6]-[11].

Some examples of log-convex interference functions are as follows.

Example 1: The linear function (12) is a log-convex interference function in the sense of Definition 8.

Example 2: The coefficients $v$ in (12) can adapt to the current interference situation. An example is the "worst-case interference"

$$
\mathcal{I}_{k}(\boldsymbol{p})=\max _{c_{k} \in \mathcal{C}_{k}} \boldsymbol{p}^{T} \boldsymbol{v}_{k}\left(c_{k}\right), \quad k \in \mathcal{K} .
$$

The parameter $c_{k}$ can stand for some uncertainty, chosen from a compact uncertainty set $\mathcal{C}_{k}$. Such worst-case interference functions are used, e.g., in the context of robust power control [41], [42]. The function (19) is a log-convex interference function.

Example 3: It was shown in [44] that any convex interference function is log-convex in the sense of Definition 8 . That is, if $\mathcal{I}(\boldsymbol{p})$ is convex, then $\mathcal{I}\left(\mathrm{e}^{\boldsymbol{s}}\right)$ is log-convex. The converse is not true, however. Therefore, the class of log-convex interference functions is broader than the class of convex interference functions. Special cases of convex interference functions include the 


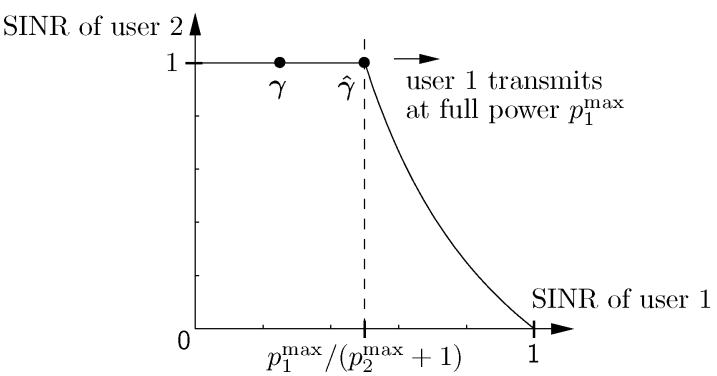

Fig. 3. Feasible SINR region for the 2-user MAC channel described in Example 4.

linear function (12) and the worst-case function (19). Hence, the requirement of log-convexity is relatively weak in comparison to many other existing interference models.

If the interference functions are linear, e.g., $\mathcal{I}(\boldsymbol{p})=\boldsymbol{v}^{T} \boldsymbol{p}$, with coupling coefficients $\boldsymbol{v} \geq 0$, and with an unconstrained power set $\mathbb{R}_{++}^{K}$, then the resulting SINR region is known to be log-convex. This was shown in [5], and extended in [6]-[8]. Recent work [4] provides conditions under which the transformed set is strictly convex, as required by Nash bargaining. However, all these results are restricted to linear interference functions. In this paper we consider the more general interference framework A1-A4 (see Definition 7). For this model, convexity of the SINR region on a logarithmic scale was shown in [3]. However, the characterization of Pareto optimality and strict convexity is still an open problem, which will be addressed in the following sections.

\section{SINR REGION WITH INDIVIDUAL POWER CONSTRAINTS}

Consider log-convex interference functions and individual power limits $\boldsymbol{p}^{\text {max }}$. Let $\boldsymbol{\gamma}>0$ be any boundary point of the resulting region $\mathcal{S}\left(\mathcal{I}, p^{\max }\right)$. The set of all power vectors achieving $\gamma$ is

$$
\mathcal{P}\left(\boldsymbol{\gamma}, \boldsymbol{p}^{\max }\right)=\left\{\mathbf{0} \leq \boldsymbol{p} \leq \boldsymbol{p}^{\max }: p_{k} \geq \gamma_{k} \mathcal{I}_{k}(\underline{\boldsymbol{p}})\right\} .
$$

For the following analysis, it is important to note that the set $\mathcal{P}\left(\boldsymbol{\gamma}, \boldsymbol{p}^{\max }\right)$ can contain multiple elements. This is most easily explained by an example, as follows.

Example 4: Consider a 2-user Gaussian multiple access channel (MAC) with successive interference cancellation, normalized noise $\sigma_{n}^{2}=1$, and a given decoding order 1,2 . The SINR of the users are

$$
\begin{aligned}
& \operatorname{SINR}_{1}(\boldsymbol{p})=\frac{p_{1}}{p_{2}+1}, \\
& \operatorname{SINR}_{2}(\boldsymbol{p})=p_{2} .
\end{aligned}
$$

Assuming power constraints $p_{1} \leq p_{1}^{\max }=1$ and $p_{2} \leq p_{2}^{\max }=$ 1, we obtain an SINR region as depicted in Fig. 3.

\section{A. Properties of Boundary Points}

Consider the boundary point $\boldsymbol{\gamma}$ depicted in Fig. 3. This point is achieved by $\boldsymbol{p}^{*}=\left[p_{1}^{\max } / 2, p_{2}^{\max }\right]^{T}$, and therefore $\boldsymbol{p}^{*} \in \mathcal{P}\left(\boldsymbol{\gamma}, \boldsymbol{p}^{\max }\right)$. This vector achieves $\boldsymbol{\gamma}$ with component-wise minimum power. However, $\boldsymbol{p}^{*}$ is not the only element of $\mathcal{P}\left(\boldsymbol{\gamma}, \boldsymbol{p}^{\max }\right)$. Because of the assumed interference cancellation, we can increase the power (and thus the SINR) of User 1, without reducing the SINR at User 2. If both users transmit with maximum power $\boldsymbol{p}^{\text {max }}$ then the corner point $\hat{\gamma}$ is achieved. This power vector is also contained in $\mathcal{P}\left(\boldsymbol{\gamma}, \boldsymbol{p}^{\max }\right)$ because $\hat{\gamma} \geq \boldsymbol{\gamma}$, so the SINR targets $\boldsymbol{\gamma}$ are still fulfilled.

The following fixed point iteration will play an important role in our analysis.

$$
p_{k}^{(n+1)}=\gamma_{k} \mathcal{I}_{k}\left(\underline{\boldsymbol{p}}^{(n)}\right), \quad \forall k \in \mathcal{K}, \quad \boldsymbol{p}^{(0)} \in \mathcal{P}\left(\boldsymbol{\gamma}, \boldsymbol{p}^{\max }\right) .
$$

Lemma 1: Let $\boldsymbol{\gamma}>0$ be an arbitrary boundary point, then the limit $\boldsymbol{p}^{*}=\lim _{n \rightarrow \infty} \boldsymbol{p}^{(n)}>0$ achieves $\boldsymbol{\gamma}$ with component-wise minimum power. That is, $\boldsymbol{p}^{*} \leq \boldsymbol{p}$ for all $\boldsymbol{p} \in \mathcal{P}\left(\boldsymbol{\gamma}, \boldsymbol{p}^{\max }\right)$.

Proof: This lemma follows from [30]. A proof for the interference framework A1-A4 was given in [43].

The next lemma shows that the inequality constraint in (20) is always fulfilled with equality for at least one component, otherwise $\boldsymbol{\gamma}$ could not be a boundary point.

Lemma 2: For any boundary point $\boldsymbol{\gamma}>0$, consider an arbitrary $\boldsymbol{p} \in \mathcal{P}\left(\boldsymbol{\gamma}, \boldsymbol{p}^{\max }\right)$. There always exists a $k \in \mathcal{K}$ such that $p_{k}=\gamma_{k} \mathcal{I}_{k}(\boldsymbol{p})$.

Proof: The proof is by contradiction. Suppose $p_{k}>$ $\gamma_{k} \mathcal{I}_{k}(\underline{\boldsymbol{p}})$ for all $k \in \mathcal{K}$. Then

$$
\begin{aligned}
C\left(\boldsymbol{\gamma}, \mathcal{I}, \boldsymbol{p}^{\max }\right) & =\inf _{\mathbf{0}<\tilde{\boldsymbol{p}} \leq \boldsymbol{p}_{\max }}\left(\max _{k \in \mathcal{K}} \frac{\gamma_{k} \mathcal{I}_{k}(\underline{\tilde{\boldsymbol{p}}})}{\tilde{p}_{k}}\right) \\
& \leq \max _{k \in \mathcal{K}} \frac{\gamma_{k} \mathcal{I}_{k}(\underline{\boldsymbol{p}})}{p_{k}}<1 .
\end{aligned}
$$

This is a contradiction because for any boundary point $\boldsymbol{\gamma}$ we have $C\left(\boldsymbol{\gamma}, \mathcal{I}, \boldsymbol{p}^{\max }\right)=1$.

Sometimes we can find a power vector such that one or more components of $\gamma$ are surpassed, as illustrated in Fig. 3. The corresponding indices are collected in an index set $\overline{\mathcal{K}}$ defined as follows.

Definition 9: For any boundary point $\gamma>0$, let $\overline{\mathcal{K}}$ be the set of all $k \in \mathcal{K}$ such that there exists a $\boldsymbol{p}^{(k)} \in \mathcal{P}\left(\boldsymbol{\gamma}, \boldsymbol{p}^{\max }\right)$ with $p_{k}^{(k)}>\gamma_{k} \mathcal{I}_{k}\left(\underline{\boldsymbol{p}}^{(k)}\right)$.

For the point $\gamma$, in Fig. 3, this is the first user, whose power can be increased without decreasing the performance of User 2 . We are only interested in the case where $\overline{\mathcal{K}}$ is nonempty. Otherwise the fixed point is the unique solution, which is trivial. Also, we know from Lemma 2 that $\overline{\mathcal{K}} \neq \mathcal{K}$.

The next theorem shows that there always exists a vector $\hat{\boldsymbol{p}}$ for which strict inequality holds for all $k \in \overline{\mathcal{K}}$ simultaneously.

Theorem 2: Let $\mathcal{I}_{1}, \ldots, \mathcal{I}_{K}$ be log-convex interference functions. Assume that $\gamma$ is an arbitrary boundary point such that $\overline{\mathcal{K}}$ is nonempty. Then there exists a vector $\hat{\boldsymbol{p}} \in \mathcal{P}\left(\boldsymbol{\gamma}, \boldsymbol{p}^{\max }\right)$ such that

$$
\hat{p}_{k}>\gamma_{k} \mathcal{I}_{k}(\underline{\hat{\boldsymbol{p}}}), \quad \forall k \in \overline{\mathcal{K}}
$$


and for all $\boldsymbol{p} \in \mathcal{P}\left(\boldsymbol{\gamma}, \boldsymbol{p}^{\max }\right)$ we have

$$
p_{k}=\gamma_{k} \mathcal{I}_{k}(\underline{\boldsymbol{p}}), \quad \forall k \in \mathcal{K} \backslash \overline{\mathcal{K}} .
$$

Proof: Inequality (24) is a consequence of Definition 9. In order to show (23), consider arbitrary $k_{1}, k_{2} \in \overline{\mathcal{K}}$, with $k_{1} \neq k_{2}$, and vectors $\boldsymbol{p}^{\left(k_{1}\right)}, \boldsymbol{p}^{\left(k_{2}\right)}$ as in Definition 9. We define a vector $\boldsymbol{p}(\lambda)$ with components

$$
p_{l}(\lambda)=\left(p_{l}^{\left(k_{1}\right)}\right)^{1-\lambda} \cdot\left(p_{l}^{\left(k_{2}\right)}\right)^{\lambda}, \quad l \in \mathcal{K} .
$$

Log-convexity implies (18). Since $\boldsymbol{p}(\lambda) \leq \boldsymbol{p}^{\max }$, we have

$$
\frac{\gamma_{l} \mathcal{I}_{l}(\underline{\boldsymbol{p}}(\lambda))}{p_{l}(\lambda)} \leq\left(\frac{\gamma_{l} \mathcal{I}_{l}\left(\underline{\boldsymbol{p}}^{\left(k_{1}\right)}\right)}{p_{l}^{\left(k_{1}\right)}}\right)^{1-\lambda} \cdot\left(\frac{\gamma_{l} \mathcal{I}_{l}\left(\underline{\boldsymbol{p}}^{\left(k_{2}\right)}\right)}{p_{l}^{\left(k_{2}\right)}}\right)^{\lambda} \leq 1 .
$$

The last inequality holds because $\boldsymbol{p}^{\left(k_{1}\right)} \in \mathcal{P}\left(\boldsymbol{\gamma}, \boldsymbol{p}^{\max }\right)$ implies that $p_{l}^{\left(k_{1}\right)} \geq \gamma_{l} \mathcal{I}_{l}\left(\underline{\boldsymbol{p}}^{\left(k_{1}\right)}\right)$, and the same holds for $\boldsymbol{p}^{\left(k_{1}\right)}$. It can be observed from (25) that $\boldsymbol{p}(\lambda) \in \mathcal{P}\left(\boldsymbol{\gamma}, \boldsymbol{p}^{\max }\right)$ for $0<\lambda<1$. For indices $l=k_{1}$ or $l=k_{2}$, at least one factor on the right hand side of (25) is strictly less than one, and therefore

$$
\frac{\gamma_{l} \mathcal{I}_{l}(\underline{\boldsymbol{p}}(\lambda))}{p_{l}(\lambda)}<1, \quad l=k_{1}, k_{2} .
$$

In the same way, we can combine $\boldsymbol{p}(1 / 2)$ with another vector $\boldsymbol{p}^{\left(k_{3}\right)}$ with $k_{3} \in \overline{\mathcal{K}}$. This leads to a new vector $\boldsymbol{p}^{\prime}(\lambda)$ which fulfills

$$
\frac{\gamma_{l} \mathcal{I}_{l}\left(\underline{\boldsymbol{p}}^{\prime}(\lambda)\right)}{p_{l}^{\prime}(\lambda)}<1, \quad l=k_{1}, k_{2}, k_{3}
$$

Repeating this procedure for all $k \in \overline{\mathcal{K}}$, we obtain the desired vector $\hat{\boldsymbol{p}} \in \mathcal{P}\left(\boldsymbol{\gamma}, \boldsymbol{p}^{\max }\right)$ fulfilling (23).

The following corollary is an immediate consequence of Theorem 2.

Corollary 2: Let $\boldsymbol{p}^{*}$ be the fixed point defined in Lemma 1 . All other quantities are defined as in Theorem 2. We have $\hat{\boldsymbol{p}} \geq \boldsymbol{p}^{*}$ (Lemma 1) and thus for all $k \in \overline{\mathcal{K}}$

$$
\hat{p}_{k}>\gamma_{k} \mathcal{I}_{k}(\underline{\hat{\boldsymbol{p}}}) \geq \gamma_{k} \mathcal{I}_{k}\left(\underline{\boldsymbol{p}}^{*}\right)=p_{k}^{*} .
$$

That is, the inequality $\hat{\boldsymbol{p}} \geq \boldsymbol{p}^{*}$ is strict for all components from $\overline{\mathcal{K}}$. In the following we will refer to $\overline{\mathcal{K}}$ as "oversized users."

The following theorem shows that the oversized users $\overline{\mathcal{K}}$ have no impact on the interference experienced by the other users $\mathcal{K} \backslash \overline{\mathcal{K}}$. That is, the interference is the same as if we would use the minimum-power vector $\boldsymbol{p}^{*}$. Also, the powers of users $\mathcal{K} \backslash \overline{\mathcal{K}}$ cannot be oversized.

Theorem 3: Let $\mathcal{I}_{1}, \ldots, \mathcal{I}_{K}$ and $\gamma$ be defined as in Theorem 2. Consider an arbitrary $\hat{\boldsymbol{p}} \in \mathcal{P}\left(\boldsymbol{\gamma}, \boldsymbol{p}^{\max }\right)$. For all $k \in \mathcal{K} \backslash \overline{\mathcal{K}}$, we have $\mathcal{I}_{k}\left(\underline{\boldsymbol{p}}^{*}\right)=\mathcal{I}_{k}(\underline{\hat{\boldsymbol{p}}})$ and $p_{k}^{*}=\hat{p}_{k}$.

Proof: We are interested in the nontrivial case $\hat{\boldsymbol{p}} \neq \boldsymbol{p}^{*}$. Defining $\boldsymbol{\Gamma}=\operatorname{diag}(\boldsymbol{\gamma})$, the fixed point iteration (21) can be written as $\boldsymbol{p}^{(n+1)}=\boldsymbol{\Gamma} \mathcal{I}\left(\boldsymbol{p}^{(n)}\right)$. By choosing the initialization $\boldsymbol{p}^{(0)}=\hat{\boldsymbol{p}}$, we obtain a monotonically decreasing sequence [30]. Since $\boldsymbol{p}^{(0)} \geq \boldsymbol{p}^{(1)}$, we have

$$
\boldsymbol{p}^{(1)}=\boldsymbol{\Gamma} \mathcal{I}\left(\underline{\boldsymbol{p}}^{(0)}\right) \geq \boldsymbol{\Gamma \mathcal { I }}\left(\underline{\boldsymbol{p}}^{(1)}\right) .
$$

Thus $\boldsymbol{p}^{(1)} \in \mathcal{P}\left(\boldsymbol{\gamma}, \boldsymbol{p}^{\max }\right)$. For any $k \in \mathcal{K} \backslash \overline{\mathcal{K}}$ we have

$$
p_{k}^{(1)}=\gamma_{k} \mathcal{I}_{k}\left(\underline{\boldsymbol{p}}^{(0)}\right)=p_{k}^{(0)}=\hat{p}_{k} .
$$

Likewise, we have for $\boldsymbol{p}^{(2)}, k \in \mathcal{K} \backslash \overline{\mathcal{K}}$

$$
p_{k}^{(2)}=\gamma_{k} \mathcal{I}_{k}\left(\underline{\boldsymbol{p}}^{(1)}\right)=p_{k}^{(1)}=\hat{p}_{k} .
$$

By induction, we have for all $n \in \mathbb{N}$

$$
p_{k}^{(n)}=\hat{p}_{k} \quad \text { for all } k \in \mathcal{K} \backslash \overline{\mathcal{K}} .
$$

With $\lim _{n \rightarrow \infty} \boldsymbol{p}^{(n)}=\boldsymbol{p}^{*}$ we have

$$
p_{k}^{*}=\hat{p}_{k} \quad \text { for all } k \in \mathcal{K} \backslash \overline{\mathcal{K}},
$$

thus proving the second statement. From the definition of $\overline{\mathcal{K}}$, we have

$$
\gamma_{k} \mathcal{I}_{k}(\underline{\hat{\boldsymbol{p}}})=\hat{p}_{k}=p_{k}^{*}=\gamma_{k} \mathcal{I}_{k}\left(\underline{\boldsymbol{p}}^{*}\right), \quad \text { for all } k \in \mathcal{K} \backslash \overline{\mathcal{K}}
$$

which concludes the proof.

Corollary 3: Consider an arbitrary $\hat{\boldsymbol{p}} \in \mathcal{P}\left(\boldsymbol{\gamma}, \boldsymbol{p}^{\max }\right)$. Then for all $\boldsymbol{p}$ with $\boldsymbol{p}^{*} \leq \boldsymbol{p} \leq \hat{\boldsymbol{p}}$ we have

$$
\mathcal{I}_{k}\left(\underline{\boldsymbol{p}}^{*}\right)=\mathcal{I}_{k}(\underline{\boldsymbol{p}})=\mathcal{I}_{k}(\underline{\hat{\boldsymbol{p}}}) \quad \text { for all } k \in \mathcal{K} \backslash \overline{\mathcal{K}} .
$$

Proof: This follows from Theorem 3 and the monotonicity axiom A3.

Note that $\boldsymbol{p}^{*}$ and $\hat{\boldsymbol{p}}$ are both contained in $\mathcal{P}\left(\boldsymbol{\gamma}, \boldsymbol{p}^{\max }\right)$, so the resulting SINR values are contained in the feasible SINR region. However, we cannot infer from Corollary 3 that the same holds for $\boldsymbol{p}$.

For $p_{k}$ from $\overline{\mathcal{K}}$ we can reduce this component without affecting the interference power. This holds for $\boldsymbol{p}^{*} \leq \boldsymbol{p} \leq \hat{\boldsymbol{p}}$ but not necessarily for vectors outside this area. This is because we cannot rely on strict monotonicity, as later in this section. We know that for $k \in \overline{\mathcal{K}}$ the interference functions for such $\boldsymbol{p}$ do not depend on the indices for which $p_{k}^{*}<\hat{p}_{k}$.

\section{B. Interference Coupling}

The structure of the SINR region depends on how the users are coupled by interference. In order to model the coupling between the axiomatic log-convex interference functions, we use the approach in [3].

We begin with the definition of the local dependency matrix, which does depend on the choice of $\boldsymbol{p}$. We define $\boldsymbol{e}_{l}$ as the allzero vector with the $l$ th component set to one.

$$
\left[\boldsymbol{D}_{\mathcal{I}}(\boldsymbol{p})\right]_{k l}= \begin{cases}1 & \begin{array}{l}
\text { if there exists a } \delta \boldsymbol{p}>0 \text { such that the } \\
\text { function } f(\delta, \boldsymbol{p})=\mathcal{I}_{k}\left(\boldsymbol{p}-\delta \boldsymbol{e}_{l}\right) \text { is } \\
\text { strictly monotonically decreasing for } \\
0 \leq \delta \leq \delta \boldsymbol{p} .
\end{array} \\
0 \quad \text { otherwise. }\end{cases}
$$


We say that an interference function $\mathcal{I}_{k}$ depends on a power $p_{l}$ if there exists an arbitrary $\boldsymbol{p}$ such that $\left[\boldsymbol{D}_{\mathcal{I}}(\boldsymbol{p})\right]_{k l}=1$. The interference coupling between users is characterized by the global dependency matrix defined as follows.

$$
\left[\boldsymbol{D}_{\mathcal{I}}\right]_{k l}= \begin{cases}1 & \text { if there exists a } \boldsymbol{p}>0 \text { such that } \\ & {\left[\boldsymbol{D}_{\mathcal{I}}(\boldsymbol{p})\right]_{k l}=1,} \\ 0 & \text { otherwise. }\end{cases}
$$

The nonzero entries in $\boldsymbol{D}_{\mathcal{I}}$ mark the transmitter/receiver pairs which are coupled by interference. A zero entry means that no interference is received, no matter how large the transmission power is. As an example, consider users that are assigned to different orthogonal resources, or separated by adaptive interference rejection techniques.

In this paper, we assume that there is no self-interference. That is, the elements of the main diagonal are zero.

Note, that this coupling model includes the widely used concept of a "link gain matrix" as a special case. In the remainder of this paper we will use $\boldsymbol{D}_{\mathcal{I}}$ in order to analyze how the interference coupling affects the structure of the boundary.

\section{Analysis of the Pareto Boundary}

Thus far, we have focused on the interference coupling aspects. Now, we will analyze QoS sets resulting from these interference models. In this paper, $Q o S$ can stand for some arbitrary performance measure, which depends on the SINR by a strictly monotone and continuous function $\phi$ defined on $\mathbb{R}_{+}$. The QoS of user $k$ is

$$
u_{k}(\boldsymbol{p})=\phi_{k}\left(\operatorname{SINR}_{k}(\boldsymbol{p})\right), \quad k \in \mathcal{K} .
$$

Common examples are MMSE, BER, delay, or capacity [43].

Let $\gamma_{k}$ be the inverse function of $\phi_{k}$, then $\gamma_{k}\left(u_{k}\right)$ is the minimum SINR level needed by the $k$ th user to satisfy the QoS target $u_{k}$. Let $\boldsymbol{u}$ be a vector of QoS values from some QoS region $\mathcal{U}$, then the associated SINR vector is

$$
\gamma(\boldsymbol{u})=\left[\gamma_{1}\left(u_{1}\right), \ldots, \gamma_{K}\left(u_{K}\right)\right]^{T} .
$$

QoS values $\boldsymbol{u}$ are feasible if and only if $\left.C\left(\boldsymbol{\gamma}(\boldsymbol{u}), \mathcal{I}, \boldsymbol{p}^{\max }\right)\right) \leq 1$. The QoS feasible set is the sublevel set

$$
\mathcal{U}=\left\{\boldsymbol{u}: C\left(\boldsymbol{\gamma}(\boldsymbol{u}), \mathcal{I}, \boldsymbol{p}^{\max }\right) \leq 1\right\} .
$$

We are now interested in the boundary of $\mathcal{U}$, which is characterized by $C\left(\boldsymbol{\gamma}(\boldsymbol{u}), \mathcal{I}, \boldsymbol{p}^{\max }\right)=1$.

Lemma 3: A boundary point $\boldsymbol{u} \in \partial \mathcal{U}$ is Pareto optimal if and only if $\boldsymbol{\gamma}(\boldsymbol{u}) \in \partial S\left(\mathcal{I}, \boldsymbol{p}^{\max }\right)$ is Pareto optimal.

Proof: This is a direct consequence of the strictly monotonic mapping (33). Pareto optimal points in $\mathcal{S}$ are mapped to Pareto optimal points in $\mathcal{U}$ and vice versa. Non-Pareto boundary segments in $\mathcal{S}$ are parallel to the coordinate axes. Those segments are mapped to parallel segments in $\mathcal{U}$ and vice versa.

From Lemma 3 it follows that we can analyze Pareto optimality of $\mathcal{U}$ by focusing on the underlying SINR set instead.

As an example, we discuss the capacity region resulting from Example 4.

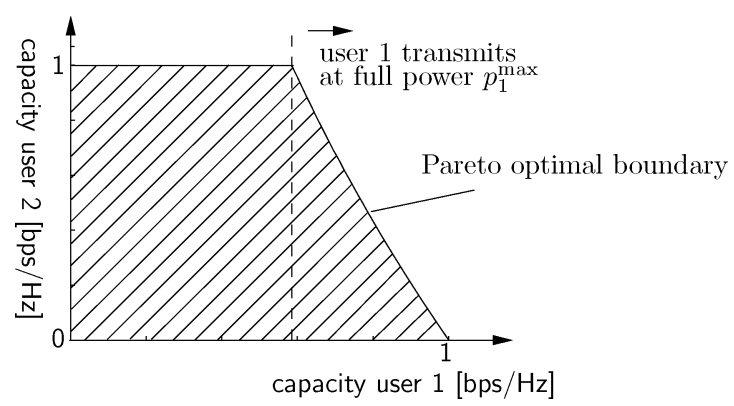

Fig. 4. Capacity region of a 2-user MAC with fixed decoding order 1, 2 and individual power limits.

Example 5: Consider the capacity region of the 2-user MAC with individual power limits as specified in Example 4. For a given decoding order 1,2, the capacities of the users are

$$
\begin{array}{ll}
\text { capacity user 1: } & C_{1}(\boldsymbol{p})=\log _{2}\left(1+\frac{p_{1}}{p_{2}+1}\right) \\
\text { capacity user 2: } & C_{2}(\boldsymbol{p})=\log _{2}\left(1+p_{2}\right) .
\end{array}
$$

Assuming that no time-sharing or rate-splitting can be performed, we obtain the capacity region depicted in Fig. 4. Note, that a part of the boundary is not Pareto optimal.

With Theorem 2 we show the following result.

Theorem 4: Consider an arbitrary boundary point $\gamma \in \partial \mathcal{S}\left(\mathcal{I}, \boldsymbol{p}^{\max }\right)$, with a fixed point $\boldsymbol{p}^{*}=\boldsymbol{p}^{*}(\boldsymbol{\gamma})$ as defined in Lemma 1. Then $\gamma$ is Pareto optimal if and only if

$$
\mathcal{P}\left(\boldsymbol{\gamma}, \boldsymbol{p}^{\max }\right)=\left\{\boldsymbol{p}^{*}\right\} .
$$

Proof: We will prove the contrapositive statement. Suppose that $\mathcal{P}\left(\boldsymbol{\gamma}, \boldsymbol{p}^{\max }\right)$ consists of multiple vectors. Then there is a nonempty set $\overline{\mathcal{K}}$ and a vector $\hat{\boldsymbol{p}}$ as in Theorem 2. For any $k \in \overline{\mathcal{K}}$ we have

$$
\hat{\gamma}_{k}=\frac{\hat{p}_{k}}{\mathcal{I}_{k}(\underline{\hat{p}})}>\gamma_{k}
$$

Thus $\hat{\gamma} \supsetneqq \gamma$, and therefore $\gamma$ is not Pareto optimal.

Conversely, assume that a boundary point $\gamma$ is not Pareto optimal, then there exists a $\tilde{\gamma}$ with $\tilde{\gamma} \supsetneqq \gamma$. This point is achieved by the power vector $\tilde{\boldsymbol{p}}$ fulfilling $\tilde{\boldsymbol{p}}=\operatorname{diag}(\tilde{\boldsymbol{\gamma}}) \mathcal{I}(\tilde{\tilde{\boldsymbol{p}}})$. We have $\tilde{\boldsymbol{p}} \neq \boldsymbol{p}^{*}$ and $\tilde{\boldsymbol{p}} \in \mathcal{P}\left(\tilde{\boldsymbol{\gamma}}, \boldsymbol{p}^{\max }\right)$. We now show that any element of $\mathcal{P}\left(\tilde{\boldsymbol{\gamma}}, \boldsymbol{p}^{\max }\right)$ is also contained in $\mathcal{P}\left(\boldsymbol{\gamma}, \boldsymbol{p}^{\max }\right)$. To this end, consider an arbitrary $\boldsymbol{p} \in \mathcal{P}\left(\tilde{\gamma}, \boldsymbol{p}^{\max }\right)$. We have

$$
p_{k} \geq \tilde{\gamma}_{k} \mathcal{I}_{k}(\underline{\boldsymbol{p}}) \geq \gamma_{k} \mathcal{I}_{k}(\underline{\boldsymbol{p}}) .
$$

Thus $\boldsymbol{p} \in \mathcal{P}\left(\boldsymbol{\gamma}, \boldsymbol{p}^{\max }\right)$, i.e., $\mathcal{P}\left(\tilde{\boldsymbol{\gamma}}, \boldsymbol{p}^{\max }\right) \subseteq \mathcal{P}\left(\boldsymbol{\gamma}, \boldsymbol{p}^{\max }\right)$. Therefore, we have determined two vectors $\tilde{\boldsymbol{p}} \neq \boldsymbol{p}^{*}$ that are both contained in $\mathcal{P}\left(\boldsymbol{\gamma}, \boldsymbol{p}^{\max }\right)$.

Next, we show how Pareto optimality is connected with the structure of the dependency matrix. To this end, consider again an arbitrary boundary point $\boldsymbol{\gamma}>0$ and $\hat{\boldsymbol{p}}$ as defined in Theorem 2. The following Lemma 4 states that if $\mathcal{P}\left(\boldsymbol{\gamma}, \boldsymbol{p}^{\max }\right)$ has multiple elements, then $\boldsymbol{D}_{\mathcal{I}}(\hat{\boldsymbol{p}})$ cannot be irreducible. A nonnegative square matrix is said to be irreducible if its directed 
graph is strongly connected. Lemma 4 will be needed later for the proof of Theorem 7 .

Lemma 4: Consider an arbitrary boundary point $\boldsymbol{\gamma} \in$ $\partial \mathcal{S}\left(\mathcal{I}, \boldsymbol{p}^{\max }\right)$, with a fixed point $\boldsymbol{p}^{*}=\boldsymbol{p}^{*}(\boldsymbol{\gamma})$ as defined in Lemma 1. If $\mathcal{P}\left(\boldsymbol{\gamma}, \boldsymbol{p}^{\max }\right) \neq\left\{\boldsymbol{p}^{*}\right\}$, then for any $\hat{\boldsymbol{p}}$ defined in Theorem 2, the local dependency matrix $\boldsymbol{D}_{\mathcal{I}}(\hat{\boldsymbol{p}})$ is reducible.

Proof: The proof is by contradiction. Suppose that $\boldsymbol{D}_{\mathcal{I}}(\hat{\boldsymbol{p}})$ is irreducible. Assumption $\mathcal{P}\left(\boldsymbol{\gamma}, \boldsymbol{p}^{\max }\right) \neq\left\{\boldsymbol{p}^{*}\right\}$ implies the existence of an oversized user. Consequently, there are two complementary sets $\overline{\mathcal{K}}$ and $\mathcal{K} \backslash \overline{\mathcal{K}}$. Because of irreducibility there exists a connecting path between both sets. That is, there exist indices $k_{1} \in \mathcal{K} \backslash \overline{\mathcal{K}}$ and $k_{2} \in \overline{\mathcal{K}}$ such that

$$
\left[\boldsymbol{D}_{\mathcal{I}}(\hat{\boldsymbol{p}})\right]_{k_{1} k_{2}}>0
$$

We can reduce the power $\hat{p}_{k_{2}}$ of the oversized user without violating the feasibility condition. That is, there exists a $\delta>0$ and a $\hat{p}_{k_{2}}^{(\delta)}=\hat{p}_{k_{2}}-\delta$ such that

$$
\hat{p}_{k_{2}}>\hat{p}_{k_{2}}^{(\delta)}>\gamma_{k_{2}} \mathcal{I}_{k_{2}}(\underline{\hat{\boldsymbol{p}}}) \text {. }
$$

By keeping all the other components $l \neq k_{2}$ fixed, we obtain a new vector $\hat{\boldsymbol{p}}^{(\delta)} \supsetneqq \hat{\boldsymbol{p}}$. Because of monotonicity A3 we have $\mathcal{I}_{k_{2}}\left(\underline{\hat{\boldsymbol{p}}}^{(\delta)}\right) \leq \mathcal{I}_{k_{2}}(\underline{\hat{\boldsymbol{p}}})$, and with (38) we know that $\hat{\boldsymbol{p}}^{(\delta)} \in$ $\mathcal{P}\left(\boldsymbol{\gamma}, \boldsymbol{p}^{\max }\right)$.

From (37) we know that by reducing the power of user $k_{2}$ we reduce the interference of user $k_{1}$. Thus

$$
\mathcal{I}_{k_{1}}\left(\underline{\hat{\boldsymbol{p}}}^{(\delta)}\right)<\mathcal{I}_{k_{1}}(\underline{\hat{\boldsymbol{p}}}), \quad k_{1} \in \mathcal{K} \backslash \overline{\mathcal{K}} .
$$

This contradicts (24) from Theorem 2, thus concluding the proof.

\section{Concept of Strongly Coupled Users}

We will now introduce the new concept of strongly coupled users. This will prove useful in further characterizing the Pareto optimal boundary. It will turn out (Theorem 7) that this is an equivalent way of expressing Pareto optimality.

Definition 10: A $K$-user system is said to be strongly coupled with power limits $\boldsymbol{p}^{\max }$, if for any point $\gamma$, for which there is a $\boldsymbol{p} \in \mathcal{P}\left(\boldsymbol{\gamma}, \boldsymbol{p}^{\max }\right)$ with

$$
\Gamma \mathcal{I}(\underline{p}) \varsubsetneqq p
$$

there exists a vector $\hat{\boldsymbol{p}}<\boldsymbol{p}$ such that

$$
\Gamma \mathcal{I}(\underline{\hat{p}})<\hat{p}
$$

The condition (40) reflects a practically relevant property: If it is possible to fulfill SINR requirements $\gamma_{1}, \ldots, \gamma_{K}$, and one user gets more than required, then all users are strongly coupled if and only if all users benefit from reducing the oversized user's power. This is an important aspect of "fairness" because it provides a mechanism for trading off resources between users.

Theorem 5: If interference functions $\mathcal{I}_{1}, \ldots, \mathcal{I}_{K}$ with power limits $\boldsymbol{p}^{\max }$ are strongly coupled, then every boundary point $\gamma$ is Pareto optimal.

Proof: Assume that $\mathcal{I}_{1}, \ldots, \mathcal{I}_{K}$ are strongly coupled. The proof is by contradiction. Suppose that there is a boundary point $\gamma$ that is not Pareto optimal. Then there is a $\hat{\gamma} \geq \boldsymbol{\gamma}$ such that we can find a $k_{0}$ with $\hat{\gamma}_{k_{0}}>\gamma_{k_{0}}$. Consider the indicator function $C$, as defined by (22). Because $C\left(\hat{\gamma}, \mathcal{I}, \boldsymbol{p}^{\max }\right)=1$, the vector $\hat{\gamma}$ is associated with a $\hat{\boldsymbol{p}}=\hat{\boldsymbol{p}}(\boldsymbol{\gamma})$ such that $\hat{\boldsymbol{p}}=\hat{\boldsymbol{\Gamma}} \mathcal{T}(\underline{\hat{\boldsymbol{p}}})$. Therefore

$$
\hat{p}_{k_{0}}=\hat{\gamma}_{k_{0}} \mathcal{I}_{k_{0}}(\underline{\hat{\boldsymbol{p}}})>\gamma_{k_{0}} \mathcal{I}_{k_{0}}(\underline{\hat{\boldsymbol{p}}}) \text {. }
$$

Because the interference functions are strongly coupled by assumption, there is a $\boldsymbol{p}<\hat{\boldsymbol{p}} \leq \boldsymbol{p}^{\max }$ such that

$$
p_{k}>\gamma_{k} \mathcal{I}_{k}(\underline{\boldsymbol{p}}) \quad k \in \mathcal{K} \text {. }
$$

This would imply

$$
C\left(\boldsymbol{\gamma}, \mathcal{I}, \boldsymbol{p}^{\max }\right) \leq \max _{k \in \mathcal{K}} \frac{\gamma_{k} \mathcal{I}_{k}(\underline{\boldsymbol{p}})}{p_{k}}<1
$$

which is a contradiction. Thus, every boundary point is Pareto optimal.

An obvious question is: does the converse of Theorem 5 hold? That is, does a Pareto optimal boundary imply a strongly coupled system? This will be shown in Section IV-F under the additional assumption of strict monotonicity. Without strict monotonicity we only have the following result.

Theorem 6: Assume that every boundary point is Pareto optimal. Consider an arbitrary point $\boldsymbol{\gamma}>0$. If there exists a $\boldsymbol{p} \leq$ $\boldsymbol{p}^{\max }$ such that

$$
\frac{p_{k}}{\mathcal{I}_{k}(\underline{\boldsymbol{p}})} \geq \gamma_{k} \quad \forall k \in \mathcal{K}
$$

with strict inequality for at least one component, then there exists a $\tilde{\boldsymbol{p}}<\boldsymbol{p}^{\max }$ with

$$
\frac{\tilde{p}_{k}}{\mathcal{I}_{k}(\underline{\tilde{\boldsymbol{p}}})}>\gamma_{k} \quad \forall k \in \mathcal{K}
$$

Proof: From assumption (44) it follows that $\gamma$ is contained in the feasible region. However, it cannot be a boundary point because of the assumed strict inequality for one component. This would contradict the assumption of Pareto optimality. Thus, $\boldsymbol{\gamma}$ must be contained in the interior of the region, for which $C\left(\boldsymbol{\gamma}, \mathcal{I}, \boldsymbol{p}^{\max }\right)<1$. So there exists a vector $\tilde{\boldsymbol{p}} \leq \boldsymbol{p}^{\max }$ that fulfills the fixed point equation

$$
\tilde{p}_{k}=\lambda \cdot \gamma_{k} \mathcal{I}_{k}(\underline{\tilde{\boldsymbol{p}}})>\gamma_{k} \mathcal{I}_{k}(\underline{\tilde{\boldsymbol{p}}}), \quad k \in \mathcal{K}
$$

where $1<\lambda=1 / C\left(\boldsymbol{\gamma}, \mathcal{I}, \boldsymbol{p}^{\max }\right)$.

Note that Theorem 6 is not the converse of Theorem 5. The result only holds for interior points, not for the boundary. In the following Section IV-F we will make the additional assumption of strict monotonicity. Under this additional condition the converse will be shown. Also, the connection with the dependency matrix $\boldsymbol{D}_{\mathcal{I}}$ will be explained.

\section{E. Strict Monotonicity on the Dependency Set}

We now introduce the additional property of strict monotonicity on the dependency set. The definition of the dependency set is based on the dependency matrix $\boldsymbol{D}_{\mathcal{I}}$. The dependency set of user $k$ is

$$
\mathrm{L}_{k}=\left\{l \in \mathcal{K}:\left[\boldsymbol{D}_{\mathcal{I}}\right]_{k l}=1\right\}
$$


This is the set of transmitters which have impact on user $k$. The set is always nonempty because axiom A1 rules out the trivial case $\mathcal{I}(\boldsymbol{p})=0, \forall \boldsymbol{p}$.

Definition 11 (Strict Monotonicity): $\mathcal{I}_{k}(\underline{\boldsymbol{p}})$ is said to be strictly monotonic (on its dependency set $\mathrm{L}_{k}$ ) if for arbitrary $\boldsymbol{p}^{(1)}, \boldsymbol{p}^{(2)}$, the inequality $\boldsymbol{p}^{(1)} \geq \boldsymbol{p}^{(2)}$, with $p_{l}^{(1)}>p_{l}^{(2)}$ for some $l \in \mathrm{L}_{k}$, implies $\mathcal{I}_{k}\left(\underline{\boldsymbol{p}}^{(1)}\right)>\mathcal{I}_{k}\left(\underline{\boldsymbol{p}}^{(2)}\right)$.

So $\mathcal{I}_{k}(\underline{p})$ is strictly increasing in at least one power component.

The assumption of strict monotonicity enables us to prove the converse of Theorem 5. In addition, it provides a link between the dependency matrix $\boldsymbol{D}_{\mathcal{I}}$ and Pareto optimality. This is summarized by the next theorem.

Theorem 7: Consider a $K$-user system with individual power limits $p^{\max }$ and interference functions $\mathcal{I}_{1}, \ldots \mathcal{I}_{K}$ that are strictly monotonic on their respective dependency sets. Then the following statements are equivalent:

- The system is strongly coupled (Definition 10).

- The dependency matrix $\boldsymbol{D}_{\mathcal{I}}$ is irreducible.

- Every boundary point is Pareto optimal.

Proof: We first show that the dependency matrix $\boldsymbol{D}_{\mathcal{I}}$ is irreducible if and only if every boundary point $\boldsymbol{\gamma}$ is Pareto optimal.

The first part is by contradiction. Suppose that $\boldsymbol{D}_{\mathcal{I}}$ is irreducible but some boundary point $\boldsymbol{\gamma}$ is not Pareto optimal (see Definition 4). Then $\mathcal{P}\left(\boldsymbol{\gamma}, \boldsymbol{p}^{\max }\right)$ has multiple elements and there is a vector $\hat{\boldsymbol{p}}$ as defined in Theorem 2. From Lemma 4 it follows that $\boldsymbol{D}_{\mathcal{I}}(\hat{\boldsymbol{p}})$ is reducible. However, this is a contradiction because irreducibility of $\boldsymbol{D}_{\mathcal{I}}$ implies irreducibility of $\boldsymbol{D}_{\mathcal{I}}(\hat{\boldsymbol{p}})$. This is shown as follows. The proof is again by contradiction. We need to show that $\left[\boldsymbol{D}_{\mathcal{I}}\right]_{k l}>0$ implies $\left[\boldsymbol{D}_{\mathcal{I}}(\underline{\hat{p}})\right]_{k l}>0$ for any $k, l \in \mathcal{K}$. Suppose that $\left[\boldsymbol{D}_{\mathcal{I}}(\hat{\boldsymbol{p}})\right]_{k l}=0$, then we know from (31) that $f(\delta, \hat{\boldsymbol{p}})=\mathcal{I}_{k}\left(\underline{\hat{\boldsymbol{p}}}-\delta \boldsymbol{e}_{l}\right)$ is constant for all $\delta>0$. This means that $\mathcal{I}_{k}$ does not depend on the $l$ th component, which contradicts the assumption of strict monotonicity, thus proving that $\boldsymbol{D}_{\mathcal{I}}(\hat{\boldsymbol{p}})$ is irreducible. Thus, we have shown that an irreducible dependency matrix $D_{\mathcal{I}}$ implies a Pareto optimal boundary.

Conversely, we need to show that if an arbitrary boundary point $\boldsymbol{\gamma}$ is Pareto optimal then $\boldsymbol{D}_{\mathcal{I}}$ is irreducible. The proof is by contradiction. Suppose that $\boldsymbol{D}_{\mathcal{I}}$ is reducible. Without loss of generality we can assume that $\boldsymbol{D}_{\mathcal{I}}$ has Frobenius normal form [47], with irreducible matrices $\boldsymbol{D}_{1}, \ldots, \boldsymbol{D}_{N}$ along the main diagonal. Such a canonical form can always be achieved by a symmetric permutation of rows and columns of $\boldsymbol{D}_{\mathcal{I}}$. Suppose that the first (isolated) block has a dimension $k_{1} \times k_{1}$. This means that the first $k_{1}$ interference functions do $n o t$ depend on the components $p_{k_{1}+1}, \ldots, p_{K}$. Thus, the vector $\boldsymbol{p}_{1}^{*}=\left[p_{1}^{\max }, \ldots, p_{k_{1}}^{\max }\right]^{T} \in \mathbb{R}_{++}^{k_{1}}$ leads to SINR values $\gamma_{k}^{*}=p_{k}^{\max } / \mathcal{I}_{k}\left(\boldsymbol{p}_{1}^{*}\right)$ for $1 \leq k \leq k_{1}$. We introduce the set $\mathcal{M}_{+}=\left\{\boldsymbol{p} \in \mathbb{R}_{+}^{K}: \boldsymbol{p}=\left[\left(\boldsymbol{p}_{1}^{*}\right)^{T}, p_{k_{1}+1}, \ldots, p_{K}\right]^{T}\right\}$. For arbitrary $\boldsymbol{\gamma}^{(2)}=\left[\gamma_{k_{1}+1}, \ldots, \gamma_{K}\right]^{T}>0$ we define

$$
C^{(2)}\left(\boldsymbol{\gamma}^{(2)}, \boldsymbol{p}^{\max }\right)=\inf _{\substack{\boldsymbol{p} \in \mathcal{M}_{+} \\ 0<\boldsymbol{p}^{\max }}} \max _{\boldsymbol{p}^{\max }+1 \leq k \leq K} \frac{\gamma_{k} \mathcal{I}_{k}(\underline{\boldsymbol{p}})}{p_{k}} .
$$

Consider $\hat{\boldsymbol{\gamma}}^{(2)}>0$ such that $C^{(2)}\left(\hat{\boldsymbol{\gamma}}^{(2)}, \boldsymbol{p}^{\max }\right)=1$. Because of the noise and power constraints, we know from [30] that this point is feasible, i.e., there exists a $\boldsymbol{p}^{(2)} \in \mathbb{R}_{+}^{K-k_{1}}$ with $p_{k}^{(2)} \leq$ $p_{k}^{\max }$ such that

$$
\hat{\gamma}_{k}^{(2)} \mathcal{I}_{k}\left(\left[\begin{array}{c}
\boldsymbol{p}_{1}^{*} \\
\boldsymbol{p}^{(2)} \\
\sigma_{n}^{2}
\end{array}\right]\right)=p_{k}^{(2)}, \quad k_{1}+1 \leq k \leq K .
$$

Here, $p_{1}^{*}$ acts like additional noise. The complete $K$-dimensional vector of SINR values is $\tilde{\gamma}=\left[\gamma_{1}^{*}, \ldots, \gamma_{k_{1}}^{*}, \hat{\gamma}_{k_{1}+1}^{(2)}, \ldots, \hat{\gamma}_{K}^{(2)}\right]^{T}$, and the vector achieving this point is $\check{\boldsymbol{p}}=\left[\left(\boldsymbol{p}_{1}^{*}\right)^{T},\left(\boldsymbol{p}^{(2)}\right)^{T}\right]^{T}$. Using (14) we obtain

$$
\begin{aligned}
C\left(\tilde{\boldsymbol{\gamma}}, \mathcal{I}, \boldsymbol{p}^{\max }\right) & \geq \inf _{0<\boldsymbol{p}^{\operatorname{p}} \boldsymbol{p}^{\max }} \max _{1 \leq k \leq k_{1}} \frac{\gamma_{k}^{*} \mathcal{I}_{k}(\underline{\boldsymbol{p}})}{p_{k}} \\
& =\frac{\gamma_{k}^{*} \mathcal{I}_{k}\left(\underline{\boldsymbol{p}}_{1}^{*}\right)}{p_{k}^{\max }}=1 .
\end{aligned}
$$

This can only be fulfilled with equality since we also have

$$
C\left(\tilde{\gamma}, \mathcal{I}, \boldsymbol{p}^{\max }\right) \leq \max _{1 \leq k \leq K} \frac{\tilde{\gamma}_{k} \mathcal{I}_{k}(\underline{\tilde{\boldsymbol{p}}})}{\check{p}_{k}}=1 .
$$

Thus, $C\left(\tilde{\boldsymbol{\gamma}}, \mathcal{I}, \boldsymbol{p}^{\max }\right)=1$.

Next, consider an arbitrary $\lambda$ with $0<\lambda<1$, and

$$
\tilde{\gamma}(\lambda)=\left[\gamma_{1}^{*}, \ldots, \gamma_{k_{1}}^{*}, \lambda \tilde{\gamma}_{k_{1}+1}, \ldots, \lambda \tilde{\gamma}_{K}\right]^{T} .
$$

We have $C\left(\tilde{\boldsymbol{\gamma}}(\lambda), \mathcal{I}, \boldsymbol{p}^{\max }\right) \leq C\left(\tilde{\boldsymbol{\gamma}}, \mathcal{I}, \boldsymbol{p}^{\max }\right)=1$. Similar to (49) it is shown that $C\left(\tilde{\gamma}(\lambda), \mathcal{I}, \boldsymbol{p}^{\max }\right) \geq 1$. Thus, $C\left(\tilde{\boldsymbol{\gamma}}(\lambda), \mathcal{I}, \boldsymbol{p}^{\max }\right)=1$ holds, which means that both $\tilde{\boldsymbol{\gamma}}$ and $\tilde{\boldsymbol{\gamma}}(\lambda)$ are boundary points. That is, we can minimize components of $\tilde{\gamma}$ without leaving the boundary, which contradicts the assumption of Pareto optimality.

Next, we show that the system is strongly coupled if and only if the dependency matrix $\boldsymbol{D}_{\mathcal{I}}$ is irreducible.

Assume that $\boldsymbol{D}_{\mathcal{I}}$ is irreducible. Consider an arbitrary boundary point $\boldsymbol{\gamma}$ such that there is a $\boldsymbol{p} \in \mathcal{P}\left(\boldsymbol{\gamma}, \boldsymbol{p}^{\max }\right)$ with $\boldsymbol{\Gamma \mathcal { I }}(\underline{\boldsymbol{p}}) \supsetneqq \boldsymbol{p}$. This inequality is strict for at least on component $k_{0}$, so we can decrease $p_{k_{0}}$ without violating the inequality. Because of the assumed strict monotonicity, decreasing $p_{k_{0}}$ decreases the interference of the users in the dependency set $L_{k_{0}}$. These users can in turn reduce their powers without without violating the above inequality. Irreducibility means that, in the graph of $\boldsymbol{D}_{\mathcal{I}}$ there is a path from any point to any other point. Thus, by successively decreasing the components of $\boldsymbol{p}$, we obtain a vector $\tilde{\boldsymbol{p}}$ with

$$
\frac{\tilde{p}_{k}}{\mathcal{I}_{k}(\underline{\tilde{p}})}>\gamma_{k}, \quad \text { for all } k \in \mathcal{K} \text {. }
$$

That is, the users are strongly coupled.

Having shown that irreducibility implies a strongly coupled system, it remains to show that if $\boldsymbol{D}_{\mathcal{I}}$ is reducible then the system cannot be strongly coupled. To this end, we use the 
SINR vectors $\tilde{\gamma}$ and $\tilde{\gamma}(\lambda)$ that were already introduced earlier in the proof. The point $\tilde{\boldsymbol{\gamma}}$ is achieved by $\check{\boldsymbol{p}}$, thus

$$
\begin{aligned}
& \frac{\check{p}_{k}}{\mathcal{I}_{k}(\underline{\check{\boldsymbol{p}}})}=\tilde{\gamma}_{k}>\lambda \tilde{\gamma}_{k}=\tilde{\gamma}_{k}(\lambda), \quad \text { for all } k_{1}+1 \leq k \leq K, \\
& \frac{\check{p}_{k}}{\mathcal{\mathcal { I }}_{k}(\underline{\check{\boldsymbol{p}}})}=\tilde{\gamma}_{k}(\lambda), \quad \text { for all } 1 \leq k \leq k_{1} .
\end{aligned}
$$

However, there is no vector $\boldsymbol{p}^{\prime} \leq \check{\boldsymbol{p}}$ with

$$
\frac{p_{k}^{\prime}}{\mathcal{I}_{k}\left(\underline{\boldsymbol{p}}^{\prime}\right)}>\gamma_{k}(\lambda), \quad 1 \leq k \leq K .
$$

Because then $\boldsymbol{\gamma}(\lambda)$ could not be a boundary point.

Theorem 7 will be needed in the next section.

\section{STRICt Log-CONVEXITy of SINR FEASIBLE SETS}

In this section we will address another important property, namely strict log-convexity. A connection between log-convex interference functions and log-convex SINR regions was already observed in previous work, e.g., [3]. In this section we will complement and extend previous work by adding power constraints. In particular, we will derive conditions for strict log-convexity of the SINR regions $\mathcal{S}\left(\mathcal{I}, p^{\max }\right)$ and $\mathcal{S}\left(\mathcal{I}, P_{t o t}\right)$, defined by (14) and (16), respectively. It will turn out that strict log-convexity depends on how users are coupled.

\section{A. Total Power Constraint}

Assume that the sum of all transmission powers is limited by $P_{t o t}$. The next theorem shows that the resulting SINR set is strictly convex after log-transformation.

Theorem 8: Let $\mathcal{I}_{1}, \ldots, \mathcal{I}_{K}$ be arbitrary log-convex interference functions. Then for all $0<P_{t o t}<+\infty$ the logarithmic transformation of the SINR region $\log \left(\mathcal{S}\left(\mathcal{I}, P_{t o t}\right)\right)$ is strictly convex, the entire boundary of $\mathcal{S}\left(\mathcal{I}, P_{t o t}\right)$ is Pareto optimal, and $\mathcal{S}\left(\mathcal{I}, P_{\text {tot }}\right) \in \mathcal{S T}_{c}$.

Proof: In order to show strict convexity, consider arbitrary points $\hat{\boldsymbol{q}}, \breve{\boldsymbol{q}}$, with $\hat{\boldsymbol{q}} \neq \check{\boldsymbol{q}}$, from the boundary of $\mathcal{L} \log \left(\mathcal{S}\left(\mathcal{I}, P_{t o t}\right)\right)$. This set is strictly convex if the line segment $\boldsymbol{q}(\lambda)=(1-\lambda) \hat{\boldsymbol{q}}+$ $\lambda \check{\boldsymbol{q}}$, with $\lambda \in(0,1)$, is in the interior of the region. This is shown in the SINR domain, where $\hat{\gamma}=\exp \hat{\boldsymbol{q}}$ and $\check{\boldsymbol{\gamma}}=\exp \check{\boldsymbol{q}}$ are the corresponding boundary points, with $\hat{\gamma} \neq \check{\gamma}$. The line segment is transformed to the curve (all operations are component-wise)

$$
\boldsymbol{\gamma}(\lambda)=\exp \boldsymbol{q}(\lambda)=(\hat{\boldsymbol{\gamma}})^{1-\lambda} \cdot(\check{\boldsymbol{\gamma}})^{\lambda} .
$$

A point $\boldsymbol{q}(\lambda)$ on the line segment is in the interior of $\log \left(\mathcal{S}\left(\mathcal{I}, P_{\text {tot }}\right)\right)$ if and only if $C\left(\boldsymbol{\gamma}(\lambda), P_{\text {tot }}\right)<1$. We exploit that for any $\gamma>0$ there exists a unique power vector $\boldsymbol{p}(\boldsymbol{\gamma})>0$ such that

$$
\gamma_{k} \frac{1}{C\left(\boldsymbol{\gamma}, P_{\text {tot }}\right)}=\frac{p_{k}(\boldsymbol{\gamma})}{\mathcal{I}_{k}(\underline{\boldsymbol{p}}(\boldsymbol{\gamma}))}, \quad \forall k \in \mathcal{K} .
$$

This can be shown in a similar way as in [30], by exploiting the strict monotonicity A4, and the fact that $\gamma_{k} / C\left(\boldsymbol{\gamma}, P_{t o t}\right)$ is a boundary point. Let us define $\boldsymbol{p}(\lambda)$, where $p_{k}(\lambda)=\left(\hat{p}_{k}\right)^{1-\lambda}$. $\left(\check{p}_{k}\right)^{\lambda}$, and $\hat{\boldsymbol{p}}:=\boldsymbol{p}(\hat{\boldsymbol{\gamma}}), \check{\boldsymbol{p}}:=\boldsymbol{p}(\check{\gamma})$ are the power vectors that achieve the boundary points $\hat{\boldsymbol{p}}$ and $\check{\boldsymbol{p}}$, respectively. Because of uniqueness, $\hat{\gamma} \neq \check{\gamma}$ implies $\hat{\boldsymbol{p}} \neq \check{\boldsymbol{p}}$. By exploiting log-convexity of the interference functions $\mathcal{I}_{1}, \ldots, \mathcal{I}_{K}$, we have

$$
\frac{\gamma_{k}(\lambda) \cdot \mathcal{I}_{k}(\underline{\boldsymbol{p}}(\lambda))}{p_{k}(\lambda)} \leq\left(\frac{\hat{\gamma}_{k} \cdot \mathcal{I}_{k}(\underline{\hat{\boldsymbol{p}}})}{\hat{p}_{k}}\right)^{1-\lambda} \cdot\left(\frac{\check{\gamma}_{k} \cdot \mathcal{I}_{k}(\underline{\underline{\boldsymbol{p}}})}{\check{p}_{k}}\right)^{\lambda}
$$

for all $k \in \mathcal{K}$. Combining (17) and (53), we have

$$
C\left(\boldsymbol{\gamma}(\lambda), \mathcal{I}, P_{t o t}\right) \leq\left[C\left(\hat{\boldsymbol{\gamma}}, \mathcal{I}, P_{t o t}\right)\right]^{1-\lambda} \cdot\left[C\left(\check{\boldsymbol{\gamma}}, \mathcal{I}, P_{t o t}\right)\right]^{\lambda} .
$$

Since $\hat{\gamma}$ and $\check{\gamma}$ are boundary points, we have $C\left(\hat{\gamma}, \mathcal{I}, P_{t o t}\right)=$ $C\left(\check{\boldsymbol{\gamma}}, \mathcal{I}, P_{t o t}\right)=1$, and thus

$$
C\left(\boldsymbol{\gamma}(\lambda), \mathcal{I}, P_{\text {tot }}\right) \leq 1 .
$$

It remains to show that inequality (54) is strict. Since $\hat{\boldsymbol{p}} \neq \check{\boldsymbol{p}}$, Hölder's inequality leads to

$$
\sum_{k \in \mathcal{K}} p_{k}(\lambda)<\left(\sum_{k \in \mathcal{K}}\left(\hat{p}_{k}\right)^{(1-\lambda) n}\right)^{\frac{1}{n}} \cdot\left(\sum_{k \in \mathcal{K}}\left(\check{p}_{k}\right)^{\lambda m}\right)^{\frac{1}{m}}
$$

where $1=1 / n+1 / m$. This expression is simplified by choosing $n=1 /(1-\lambda)$ and $m=1 / \lambda$. Since the sum-power constraint is active for points on the boundary, we have $P_{\text {tot }}=\sum_{k} \hat{p}_{k}=\sum_{k} \breve{p}_{k}$. Thus,

$$
\sum_{k \in \mathcal{K}} p_{k}(\lambda)<\left(\sum_{k \in \mathcal{K}} \hat{p}_{k}\right)^{1-\lambda} \cdot\left(\sum_{k \in \mathcal{K}} \check{p}_{k}\right)^{\lambda}=P_{t o t} .
$$

Since inequality (56) is strict, there exists a $\mu>1$, and a new vector $\boldsymbol{p}^{\prime}(\lambda)=\mu \boldsymbol{p}(\lambda)$ that also fulfills the inequality. By exploiting axioms $\mathrm{A} 2$ and $\mathrm{A} 4$, we have

$$
\begin{aligned}
\frac{\gamma_{k}(\lambda) \cdot \mathcal{I}_{k}\left(\underline{\boldsymbol{p}^{\prime}}(\lambda)\right)}{p_{k}^{\prime}(\lambda)} & =\frac{\gamma_{k}(\lambda) \cdot \mathcal{I}_{k}\left(\boldsymbol{p}(\lambda), \sigma_{n}^{2} / \mu\right)}{p_{k}(\lambda)} \\
& <\frac{\gamma_{k}(\lambda) \cdot \mathcal{I}_{k}\left(\boldsymbol{p}(\lambda), \sigma_{n}^{2}\right)}{p_{k}(\lambda)} .
\end{aligned}
$$

From A3 it follows that inequality (53) is strict. Thus, $C\left(\boldsymbol{\gamma}(\lambda), \mathcal{I}, P_{t o t}\right)<1$, which means that for any $\lambda \in(0,1)$, the point $\boldsymbol{q}(\lambda)$ is in the strict interior of the region, thus proving strict log-convexity of the SINR region.

Strict log-convexity implies Pareto optimality. It remains to show that $\mathcal{S}\left(\mathcal{I}, P_{\text {tot }}\right) \in \mathcal{S} \mathcal{T}_{c}$. The transformed set $\log \left(\mathcal{S}\left(\mathcal{I}, P_{\text {tot }}\right)\right)$ is closed. This can be observed from definition (16). It is also upper-bounded because of the power constraint and the assumption of noise. Finally, the entire boundary is Pareto optimal, thus $\mathcal{S}\left(\mathcal{I}, P_{t o t}\right) \in \mathcal{S} \mathcal{T}_{c}$ is fulfilled.

As a consequence of Theorem 8, the solution of (3), respectively, (2), is the single-valued NBS. The SINR region is a compact set, and therefore the existence of the unique optimizer is guaranteed.

\section{B. Individual Power Constraints}

The previous section has shown that the possible occurrence of interference-free users does not matter under a sum-power constraint, because the users are always coupled by sharing a 
common power budget. However, in order to analyze the behavior under individual power constraints, we need to take into account the interference coupling characterized by $\boldsymbol{D}_{\mathcal{I}}$.

We now study under which conditions the SINR region $\mathcal{S}\left(\mathcal{I}, p^{\max }\right)$, defined by (14), is strictly log-convex. Then, we study under which conditions the SINR region is contained in $\mathcal{S T}_{c}$.

We begin by defining strictly log-convex interference functions.

Definition 12 (Strict Log-Convexity): A log-convex interference function $\mathcal{I}_{k}$ is said to be strictly log-convex if for all $\hat{\boldsymbol{p}}, \check{\boldsymbol{p}}$ for which there is some $l \in \mathrm{L}_{k}$ with $\hat{p}_{l} \neq \check{p}_{l}$, the following inequality holds:

$$
\mathcal{I}_{k}(\underline{\boldsymbol{p}}(\lambda))<\left(\mathcal{I}_{k}(\underline{\hat{\boldsymbol{p}}})\right)^{1-\lambda} \cdot\left(\mathcal{I}_{k}(\underline{\check{\boldsymbol{p}}})\right)^{\lambda}, \quad \lambda \in(0,1) .
$$

where $\boldsymbol{p}(\lambda)=\hat{\boldsymbol{p}}^{1-\lambda} \cdot \check{\boldsymbol{p}}^{\lambda}$.

The following lemma shows that strict log-convexity implies strict monotonicity.

Lemma 5: Every strictly log-convex interference function $\mathcal{I}_{k}$ is strictly monotonic on its dependency set (see Definition 11).

Proof: Consider an arbitrary fixed vector $\boldsymbol{p} \in \mathbb{R}_{++}^{K}$, and an arbitrary $l \in \mathrm{L}_{k}$. We define

$$
\boldsymbol{p}^{(l)}(x)=\boldsymbol{p}+x \boldsymbol{e}_{l}, \quad x>0
$$

and

$$
\boldsymbol{p}(\lambda)=(\boldsymbol{p})^{1-\lambda} \cdot\left(\boldsymbol{p}^{(l)}(x)\right)^{\lambda}, \quad \lambda \in(0,1) .
$$

Since $l \in \mathrm{L}_{k}$, strict log-convexity implies

$$
\mathcal{I}_{k}(\underline{\boldsymbol{p}}(\lambda))<\left(\mathcal{I}_{k}(\underline{\boldsymbol{p}})\right)^{1-\lambda} \cdot\left(\mathcal{I}_{k}\left(\underline{\boldsymbol{p}}^{(l)}(x)\right)\right)^{\lambda} .
$$

By (60) we have

$$
p_{v}(\lambda)=p_{v} \text { for all } v \neq l .
$$

Also, $x>0$ implies

$$
p_{l}(\lambda)=\left(p_{l}\right)^{1-\lambda} \cdot\left(p_{l}+x\right)^{\lambda}>p_{l} .
$$

With A3 (monotonicity) we know that $\boldsymbol{p} \leq \boldsymbol{p}(\lambda)$ implies $\mathcal{I}_{k}(\underline{\boldsymbol{p}}) \leq \mathcal{I}_{k}(\underline{\boldsymbol{p}}(\lambda))$. With (61) we have

$$
\mathcal{I}_{k}(\underline{\boldsymbol{p}})<\left(\mathcal{I}_{k}(\underline{\boldsymbol{p}})\right)^{1-\lambda} \cdot\left(\mathcal{I}_{k}\left(\underline{\boldsymbol{p}}^{(l)}(x)\right)\right)^{\lambda}
$$

thus

$$
\left(\mathcal{I}_{k}(\underline{\boldsymbol{p}})\right)^{\lambda}<\left(\mathcal{I}_{k}\left(\underline{\boldsymbol{p}}^{(l)}(x)\right)\right)^{\lambda}
$$

which shows strict monotonicity.

Note that the converse of Lemma 5 is not true. The following example shows a strictly monotone interference function which is not strictly log-convex. That is, strict monotonicity is weaker than strict log-convexity.

Example 6: Consider the interference function

$\mathcal{I}(\boldsymbol{p})=C \cdot \prod_{k \in \mathcal{K}}\left(p_{k}\right)^{w_{k}}, \quad$ with $\sum_{l \in \mathcal{K}} w_{l}=1$ and $\min _{l \in \mathcal{K}} w_{l}>0$.
Using the same notation as in Definition 12 we have $\mathcal{I}(\boldsymbol{p}(\lambda))=$ $\mathcal{I}(\hat{\boldsymbol{p}})^{1-\lambda} \cdot \mathcal{I}(\check{\boldsymbol{p}})^{\lambda}$. Thus, (65) is log-convex but not strictly logconvex. However, (65) is strictly monotone.

Under the assumption of strict log-convexity we can derive a necessary and sufficient condition for strict convexity of the transformed SINR region. To this end, we need the following result.

Lemma 6: Let $\mathcal{I}_{1}, \ldots \mathcal{I}_{K}$ be strictly log-convex interference functions, and each user affects the interference function of at least one other user, i.e., each column of $\boldsymbol{D}_{\mathcal{I}}$ has at least one nonzero entry off the main diagonal. Then for arbitrary $\hat{\boldsymbol{p}} \neq \check{\boldsymbol{p}}$ there exists at least one $k_{0}$ such that

$$
\mathcal{I}_{k_{0}}(\underline{\boldsymbol{p}}(\lambda))<\left(\mathcal{I}_{k_{0}}(\underline{\hat{\boldsymbol{p}}})\right)^{1-\lambda} \cdot\left(\mathcal{I}_{k_{0}}(\underline{\check{\boldsymbol{p}}})\right)^{\lambda} \quad \forall \lambda \in(0,1) .
$$

Proof: If (66) is fulfilled for one $\lambda_{0}$ then it is fulfilled for all $\lambda \in(0,1)$. This follows from the strict log-convexity of the interference functions. Next, we prove Lemma 6 by contradiction. Suppose that there is a $\lambda_{0} \in(0,1)$ such that for all $k \in \mathcal{K}$

$$
\mathcal{I}_{k}\left(\underline{\boldsymbol{p}}\left(\lambda_{0}\right)\right)=\left(\mathcal{I}_{k}(\underline{\hat{\boldsymbol{p}}})\right)^{1-\lambda_{0}} \cdot\left(\mathcal{I}_{k}(\underline{\check{\boldsymbol{p}}})\right)^{\lambda_{0}} \cdot
$$

This can only be fulfilled if for all $k \in \mathcal{K}$ there exists a $c_{k}>0$ such that

$$
\underline{\hat{p}}_{l}=c_{k} \underline{\check{p}}_{l} \quad \text { for all } l \in \underline{\mathrm{L}}_{k}
$$

where $\underline{\mathrm{L}}_{k}=\left(\mathrm{L}_{k}, K+1\right)$ is the extended dependency set, which always contains the noise index $K+1$. Both vectors have the same last component $\underline{\hat{p}}_{K+1}=\underline{\underline{p}}_{K+1}$, thus $c_{k}=1$ for all $k \in \mathcal{K}$. By assumption, each user depends on at least one other user, thus for each index $l$, there exists a $\tilde{k}$ such that $l \in \underline{\mathrm{L}}_{\tilde{k}}$.

Thus, we have equality for all components, which leads to the contradiction $\hat{\boldsymbol{p}}=\check{\boldsymbol{p}}$.

Note that the constant noise component $\underline{p}_{K+1}$ plays a crucial role in the proof of Lemma 6. Axiom A4 implies strict monotonicity with respect to $\underline{p}_{K+1}$. We can use Lemma 6 to derive a necessary and sufficient condition for strict log-convexity.

Theorem 9: Let $\mathcal{I}_{1}, \ldots \mathcal{I}_{K}$ be strictly log-convex interference functions. The transformed $\operatorname{SINR}$ region $\left.\log \left(\mathcal{S}\left(\mathcal{I}, \boldsymbol{p}^{\max }\right)\right)\right)$ is strictly convex if and only if $\boldsymbol{D}_{\mathcal{I}}$ is irreducible.

Proof: $\mathcal{I}_{1}, \ldots \mathcal{I}_{K}$ are strictly log-convex and thus they are also strictly monotonic on their respective dependency set (see Lemma 5). If the region is strictly convex, then the entire boundary is Pareto optimal. Theorem 7 implies that $\boldsymbol{D}_{\mathcal{I}}$ is irreducible.

It remains to show that irreducibility implies a strictly convex region. Consider arbitrary boundary points $\hat{\gamma}$ and $\check{\gamma}$ with corresponding power vectors $\hat{\boldsymbol{p}}$ and $\breve{\boldsymbol{p}}$. As in the proof of Theorem 8 , we use $\boldsymbol{\gamma}(\lambda)$ and $\boldsymbol{p}(\lambda)$. We have $\boldsymbol{p}(\lambda)=\hat{\boldsymbol{p}}^{1-\lambda} \cdot \check{\boldsymbol{p}}^{\lambda} \leq$ $\left(\boldsymbol{p}^{\max }\right)^{1-\lambda} \cdot\left(\boldsymbol{p}^{\max }\right)^{\lambda}=\boldsymbol{p}^{\max }$. In [3, Appendix B] it was shown that $\gamma_{k}(\lambda) \leq p_{k}(\lambda) / \mathcal{I}_{k}(\boldsymbol{p}(\lambda))$. We now exploit that $\boldsymbol{D}_{\mathcal{I}}$ is irreducible, thus each column of $\boldsymbol{D}_{\mathcal{I}}$ has at least one nonzero entry outside the main diagonal. From Lemma 6 we know that for any 
$\hat{\boldsymbol{p}} \neq \check{\check{\boldsymbol{p}}}$ there is at least one component $k_{0}$ such that (66) is fulfilled. It follows that there exists a $k_{0}$ such that

$$
\gamma_{k_{0}}(\lambda)<\frac{p_{k_{0}}(\lambda)}{\mathcal{I}_{k_{0}}(\underline{\boldsymbol{p}}(\lambda))}
$$

From Theorem 7 we know that the system is strongly coupled, so there exists a $\tilde{\boldsymbol{p}}(\lambda)<\boldsymbol{p}(\lambda) \leq \boldsymbol{p}^{\max }$ such that for all $k$

$$
\gamma_{k}(\lambda)<\frac{\tilde{p}_{k}(\lambda)}{\mathcal{I}_{k}(\underline{\tilde{\boldsymbol{p}}}(\lambda))}, \quad k \in \mathcal{K} .
$$

With (15) we have

$$
C\left(\boldsymbol{\gamma}(\lambda), \mathcal{I}, \boldsymbol{p}^{\max }\right) \leq \max _{k \in \mathcal{K}} \frac{\gamma_{k}(\lambda) \mathcal{I}_{k}(\underline{\tilde{\boldsymbol{p}}}(\lambda))}{\tilde{p}_{k}(\lambda)}<1
$$

Thus, $\boldsymbol{\gamma}(\lambda)$ is in the interior of the region.

Next, consider the function

$$
F_{\boldsymbol{w}}(s)=\sum_{k \in \mathcal{K}} w_{k} \log \frac{\mathcal{I}_{k}\left(\mathrm{e}^{\underline{\boldsymbol{s}}}\right)}{\mathrm{e}^{s_{k}}}
$$

where we have used the change of variable $\underline{\boldsymbol{s}}=\log \boldsymbol{p}$. The weights $w_{k}$ account for possible user priorities. Note that minimizing (69) is equivalent to maximizing $\sum_{k} \log \left(p_{k} / \mathcal{I}_{k}(\underline{\boldsymbol{p}})\right)$. For equal weights, this is a special case of the optimization problem (3), where the utility is the SINR.

Convexity of $F_{\boldsymbol{w}}(\boldsymbol{s})$ was already shown in [3]. However, in order to show that the SINR region is contained in $\mathcal{S} \mathcal{T}_{c}$, we need strict convexity.

Theorem 10: Let $\mathcal{I}_{1}, \ldots \mathcal{I}_{K}$ be defined as in Lemma 6. Then $F_{\boldsymbol{w}}(\boldsymbol{s})$ is strictly convex for all $\boldsymbol{w}>0$. That is, for all $\hat{\boldsymbol{s}} \neq \check{\boldsymbol{s}}$, we have

$$
F_{\boldsymbol{w}}(\boldsymbol{s}(\lambda))<(1-\lambda) F_{\boldsymbol{w}}(\hat{\boldsymbol{s}})+\lambda F_{\boldsymbol{w}}(\check{\boldsymbol{s}}) \quad \forall \lambda \in(0,1)
$$

where $\boldsymbol{s}(\lambda)=\log \boldsymbol{p}(\lambda)$, as defined in the proof of Theorem 8 .

Proof: Assume an arbitrary $\boldsymbol{w}>0$. For any $\lambda \in(0,1)$ there exists a $k_{0}$ such that (66) is fulfilled. Thus

$$
\begin{aligned}
& F \boldsymbol{w}(\boldsymbol{s}(\lambda))=w_{k_{0}} \log \frac{\mathcal{I}_{k_{0}}\left(\mathrm{e}^{\boldsymbol{s}(\lambda)}\right)}{\mathrm{e}^{s_{k_{0}}(\lambda)}}+\sum_{k \in \mathcal{K} \backslash k_{0}} w_{k} \log \frac{\mathcal{I}_{k}\left(\mathrm{e}^{\underline{\boldsymbol{s}}(\lambda)}\right)}{\mathrm{e}^{s_{k}(\lambda)}} \\
& <w_{k_{0}}(1-\lambda) \log \frac{\mathcal{I}_{k_{0}}\left(\mathrm{e}^{\hat{\boldsymbol{s}}}\right)}{\mathrm{e}^{\hat{s}_{k_{0}}}}+w_{k_{0}} \lambda \log \frac{\mathcal{I}_{k_{0}}\left(\mathrm{e}^{\check{\boldsymbol{s}}}\right)}{\mathrm{e}^{\check{s}_{k_{0}}}}+ \\
& +(1-\lambda) \sum_{k \in \mathcal{K} \backslash k_{0}} w_{k} \log \frac{\mathcal{I}_{k}\left(\mathrm{e}^{\underline{\hat{\boldsymbol{s}}}}\right)}{\mathrm{e}^{\hat{\boldsymbol{s}}_{k}}} \\
& +\lambda \sum_{k \in \mathcal{K} \backslash k_{0}} w_{k} \log \frac{\mathcal{I}_{k}\left(\mathrm{e}^{\check{\check{s}}}\right)}{\mathrm{e}^{\check{s}_{k}}} \\
& =(1-\lambda) F \boldsymbol{w}(\hat{\boldsymbol{s}})+\lambda F \boldsymbol{w}(\check{\boldsymbol{s}}) \text {. }
\end{aligned}
$$

Here we have exploited (66) and log-convexity of $\mathcal{I}_{k}$.

The next corollary is an immediate consequence of Theorem 10.
Corollary 4: The optimization problem

$$
\min _{\boldsymbol{s} \leq \log \boldsymbol{p}^{\max }} F_{\boldsymbol{w}}(\boldsymbol{s})
$$

has exactly one minimizer.

It was shown in Section II that for any set $\mathcal{U} \in \mathcal{S} \mathcal{T}_{c}$ the properties of the classical Nash bargaining framework are preserved. The following theorem shows a sufficient condition for the SINR region $\mathcal{S}\left(\mathcal{I}, \boldsymbol{p}^{\max }\right)$ to be contained in $\mathcal{S} \mathcal{T}_{c}$. The result builds on Theorem 10.

Note that sets from $\mathcal{S} \mathcal{T}_{c}$ do not need to be strictly log-convex (see Fig. 1). Thus, irreducibility of $\boldsymbol{D}_{\mathcal{I}}$, which was required in Theorem 9, is not necessary in this case.

Theorem 11: Let $\mathcal{I}_{1}, \ldots \mathcal{I}_{K}$ be defined as in Lemma 6. Then the $\mathrm{SINR}$ region $\mathcal{S}\left(\mathcal{I}, \boldsymbol{p}^{\max }\right)$ is contained in $\mathcal{S} \mathcal{T}_{c}$.

Proof: The region is (relatively) closed and upper-bounded because of the power constraints. The image set $\mathcal{Q}=$ $\mathcal{L} \operatorname{og}\left(\mathcal{S}\left(\mathcal{I}, \boldsymbol{p}^{\max }\right)\right)$ is closed and upper-bounded. We need to show that for two arbitrary Pareto optimal boundary points $\hat{\boldsymbol{q}} \neq \check{\boldsymbol{q}}$, any point $\boldsymbol{q}(\lambda)=(1-\lambda) \hat{\boldsymbol{q}}+\lambda \check{\boldsymbol{q}}$, with $\lambda \in(0,1)$, is contained in the interior of the set. This is illustrated in Fig. 1.

The proof is by contradiction. Suppose that there is a $\lambda$ such that $\boldsymbol{q}(\lambda)$ is not in the interior. Since $\mathcal{Q}$ is convex comprehensive [3], this can only be fulfilled if

$$
C\left(\log \boldsymbol{q}(\lambda), \mathcal{I}, \boldsymbol{p}^{\max }\right)=1, \quad \forall \lambda \in(0,1)
$$

Because of (72) there exists a vector $\hat{\boldsymbol{w}}>0$ such that

$$
\boldsymbol{q}(\lambda) \in \underset{\boldsymbol{q} \in \mathcal{Q}}{\arg \max } \sum_{k \in \mathcal{K}} \hat{w}_{k} q_{k}
$$

The set of maximizers of (73) is a convex set. For every maximizer $\boldsymbol{q}(\lambda)$ there is a corresponding vector $\boldsymbol{p}(\lambda)=\exp \boldsymbol{s}(\lambda)$ which fulfills the power constraints, and $s(\lambda)$ is a solution of

$$
\boldsymbol{s}(\lambda) \in \underset{\mathbf{0}<\mathrm{e}^{\boldsymbol{s}_{\leq}} \leq \boldsymbol{p}^{\max }}{\arg \max } F_{\boldsymbol{w}}(\boldsymbol{s})
$$

That is, (74) has no unique optimum. This is a contradiction because $F_{\boldsymbol{w}}(\boldsymbol{s})$ is strictly convex.

\section{CONCLUSIONS}

In this paper we have analyzed log-convex utility regions, resulting from different assumptions and power constraints and interference coupling. This "hidden convexity" is useful for developing resource allocation strategies that operate on the boundary of the region. As an example, we have analyzed the Nash bargaining problem. We have shown that the properties of the classical Nash bargaining solution are preserved for certain nonconvex utility sets. This paper provides a theoretical basis for exploiting log-convexity in interference-coupled multiuser networks and extends previous results in [4].

The results are potentially useful for different kinds of resource allocation problems. Nash bargaining is just one possible operating point. Other types of fairness can be studied in future work. 


\section{REFERENCES}

[1] J. F. Nash Jr., "The bargaining problem," Econometr., Econometr. Soc., vol. 18, pp. 155-162, 1950.

[2] F. Kelly, A. Maulloo, and D. Tan, "Rate control for communication networks: Shadow prices, proportional fairness and stability," J. Operat. Res. Soc., vol. 49, no. 3, pp. 237-252, Mar. 1998.

[3] H. Boche and M. Schubert, "A calculus for log-convex interference functions," IEEE Trans. Inf. Theory, vol. 54, no. 12, pp. 5469-5490, Dec. 2008.

[4] H. Boche and S. Stanczak, "Strict convexity of the feasible log-SIR region," IEEE Trans. Commun., vol. 56, no. 9, pp. 1511-1518, Sep. 2008.

[5] C. W. Sung, "Log-convexity property of the feasible SIR region in power-controlled cellular systems," IEEE Commun. Lett., vol. 6, no. 6, pp. 248-249, Jun. 2002.

[6] D. Catrein, L. Imhof, and R. Mathar, "Power control, capacity, and duality of up- and downlink in cellular CDMA systems," IEEE Trans. Commun., vol. 52, no. 10, pp. 1777-1785, 2004.

[7] H. Boche and S. Stańczak, "Convexity of some feasible QoS regions and asymptotic behavior of the minimum total power in CDMA systems," IEEE Trans. Commun., vol. 52, no. 12, pp. 2190-2197, Dec. 2004.

[8] H. Boche and S. Stańczak, "Log-convexity of the minimum total power in CDMA systems with certain quality-of-service guaranteed," IEEE Trans. Inform. Theory, vol. 51, no. 1, pp. 374-381, Jan. 2005.

[9] R. M. L. Imhof, "The geometry of the capacity region for CDMA systems with general power constraints," IEEE Trans. Wireless Commun., vol. 4, no. 5, pp. 2040-2044, Sep. 2005.

[10] M. Chiang, C. W. Tan, D. Palomar, D. O'Neill, and D. Julian, "Power control by geometric programming," IEEE Trans. Wireless Commun., vol. 6, no. 7, pp. 2640-2651, Jul. 2007.

[11] C. W. Tan, D. P. Palomar, and M. Chiang, "Exploiting hidden convexity for flexible and robust resource allocation in cellular networks," in Proc. IEEE Infocom, May 2007, pp. 964-972.

[12] H. J. M. Peters, Axiomatic Bargaining Game Theory. Dordrecht, Germany: Kluwer Academic , 1992.

[13] W. Thomson, "Cooperative models of bargaining," in Handbook of Game Theory. New York: Elsevier Science, 1994, vol. 2.

[14] A. Leshem and E. Zehavi, "Bargaining over the interference channel," IEEE J. Sel. Areas Commun., vol. 26, no. 7, 2008.

[15] E. G. Larsson and E. A. Jorswieck, "The MISO interference channel: Competition versus collaboration," in Proc. Allerton Conf. on Commun., Contr. Computing, Monticello, IL, 2007.

[16] R. Mazumdar, L. G. Mason, and C. Douligeris, "Fairness in network optimal flow control: Optimality of product forms," IEEE Trans. Commun., vol. 39, no. 5, pp. 775-782, May 1991.

[17] H. Yaïche, R. Mazumdar, and C. Rosenberg, "A game theoretic framework for bandwidth allocation and pricing in broadband networks," IEEE/ACM Trans. Netw., vol. 8, no. 5, pp. 667-678, 2000.

[18] C. Touati, E. Altman, and J. Galtier, "Utility based fair bandwidth allocation," in Proc. IASTED Int. Conf. Netw., Parallel and Distrib. Process. Appl. (NPDPA), Tsukuba, Japan, Oct. 2002.

[19] Z. Han, Z. Ji, and K. J. R. Liu, "Fair multiuser channel allocation for OFDMA networks using Nash bargaining solutions and coalitions," IEEE Trans. Commun., vol. 53, no. 8, pp. 1366-1376, Aug. 2005.

[20] C. Touati, E. Altman, and J. Galtier, "Generalized Nash bargaining solution for bandwidth allocation," Comput. Netw., vol. 50, no. 17, pp. 3242-3263, 2006.

[21] J. E. Suris, L. A. DaSilva, Z. Han, and A. B. MacKenzie, "Cooperative game theory for distributed spectrum sharing," in Proc. IEEE Int. Conf. Commun. (ICC), Glasgow, Scotland, Jun. 2007.

[22] G. de Clippel, "An axiomatization of the Nash bargaining solution," Social Choice and Welfare, pp. 201-210, Sep. 2007.

[23] J.-Y. Le Boudec, "Rate Adaptation, Congestion Control and Fairness: A Tutorial," Tutorial, Tech. Rep. Ecole Polytechnique Federale de Lausanne (EPFL), 2003.

[24] C. Touati, H. Kameda, and A. Inoie, Fairness in Non-Convex Systems Univ. Tsukuba, 2005, Tech. Rep. CS-TR-05-4.
[25] J. Brehmer and W. Utschick, "On proportional fairness in nonconvex wireless systems," in Proc. Int. ITG Workshop on Smart Antennas (WSA) 2009, Berlin, Germany, Feb. 2009.

[26] L. Zhou, "The Nash bargaining theory with nonconvex problems," Econometrica, Econometric Soc., vol. 3, no. 65, pp. 681-686, May 1997.

[27] M. Kaneko, "An extension of Nash bargaining problem and the Nash social welfare function," Theory and Decision, vol. 12, no. 2, pp. 135-148, Jun. 1980.

[28] M. Herrero, “The Nash program: Non-convex bargaining problems," $J$. Econom. Theory, vol. 49, pp. 266-277, 1989.

[29] J. P. Conley and S. Wilkie, "An extension of the Nash bargaining solution to nonconvex problems," Games and Econom. Behav., vol. 13, no. 1, pp. 26-38, Mar. 1996.

[30] R. D. Yates, "A framework for uplink power control in cellular radio systems," IEEE J. Sel. Areas Commun., vol. 13, no. 7, pp. 1341-1348, Sep. 1995.

[31] C. Huang and R. Yates, "Rate of convergence for minimum power assignment algorithms in cellular radio systems," Baltzer/ACM Wireless Netw., vol. 4, pp. 223-231, 1998.

[32] K. K. Leung, C. W. Sung, W. S. Wong, and T. Lok, "Convergence theorem for a general class of power-control algorithms," IEEE Trans. Commun., vol. 52, no. 9, pp. 1566-1574, Sep. 2004.

[33] F. Rashid-Farrokhi, L. Tassiulas, and K. J. Liu, "Joint optimal power control and beamforming in wireless networks using antenna arrays," IEEE Trans. Commun., vol. 46, no. 10, pp. 1313-1323, Oct. 1998.

[34] M. Bengtsson and B. Ottersten, "Optimal and suboptimal transmit beamforming," in Handbook of Antennas in Wireless Communications. Boca Raton, FL: CRC , 2001, ch. 18.

[35] M. Schubert and H. Boche, "Solution of the multi-user downlink beamforming problem with individual SINR constraints," IEEE Trans. Veh. Technol., vol. 53, no. 1, pp. 18-28, Jan. 2004.

[36] D. Hammarwall, M. Bengtsson, and B. Ottersten, "On downlink beamforming with indefinite shaping constraints," IEEE Trans. Signal Process., vol. 54, no. 9, pp. 3566-3580, Sep. 2006.

[37] P. Kumar, R. Yates, and J. Holtzman, "Power control based on bit error (BER) measurements," in Proc. IEEE Military Commun. Conf. MILCOM 95, McLean, VA, Nov. 1995, pp. $617-620$.

[38] S. Ulukus and R. Yates, "Adaptive power control and MMSE interference suppression," ACM Wireless Netw., vol. 4, no. 6, pp. 489-496, 1998.

[39] S. Hanly, "An algorithm for combined cell-site selection and power control to maximize cellular spread spectrum capacity," IEEE J. Sel. Areas Commun., vol. 13, no. 7, pp. 1332-1340, Sep. 1995.

[40] R. Yates and H. Ching-Yao, "Integrated power control and base station assignment," IEEE Trans. Veh. Technol., vol. 44, no. 3, pp. 638-644, Aug. 1995.

[41] M. Biguesh, S. Shahbazpanahi, and A. B. Gershman, "Robust downlink power control in wireless cellular systems," EURASIP J. Wireless Commun. Netw., no. 2, pp. 261-272, 2004.

[42] M. Payaró, A. Pascual-Iserte, and M. A. Lagunas, "Robust power allocation designs for multiuser and multiantenna downlink communication systems through convex optimization," IEEE Sel. Areas Commun., vol. 25, no. 7, pp. 1390-1401, Sep. 2007.

[43] M. Schubert and H. Boche, "QoS-based resource allocation and transceiver optimization," Found. Trends in Commun. Inf. Theory, vol. 2, no. 6, 2005/2006

[44] H. Boche and M. Schubert, "A unifying approach to interference modeling for wireless networks," IEEE Trans. Signal Process., vol. 58, no. 6, pp. 3282-8297, Jun. 2010.

[45] H. Boche and M. Schubert, "The structure of general interference functions and applications," IEEE Trans. Inf. Theory, vol. 54, no. 11, pp. 4980-4990, Nov. 2008.

[46] S. Boyd and L. Vandenberghe, Convex Optimization. Cambridge, U.K.: Cambridge Univ. Press, 2004.

[47] F. R. Gantmacher, The Theory of Matrices. New York: Chelsea, 1959, vol. 2. 
Holger Boche (M'04-SM'07) received the Dipl.-Ing. and Dr.-Ing. degrees in electrical engineering from the Technische Universität Dresden, Germany, in 1990 and 1994, respectively. In 1992, he graduated in mathematics from the Technische Universität Dresden, and received the Dr. rer. nat. degree in pure mathematics from the Technische Universität Berlin, Germany, in 1998.

From 1994 to 1997, he did postgraduate studies in mathematics at the Friedrich-Schiller Universität Jena, Germany. In 1997, he joined the Heinrich-Hertz-Institut (HHI) für Nachrichtentechnik Berlin. He is Head of the Broadband Mobile Communication Networks department at HHI. Since 2002, he has been Full Professor for Mobile Communication Networks at the Technische Universität Berlin at the Institute for Communications Systems, and since 2003, has been the Director of the Fraunhofer German-Sino Lab for Mobile Communications, Berlin. He was a visiting professor with the ETH Zurich, Switzerland, during winter terms 2004 and 2006 and with KTH Stockholm during summer term 2005.

Professor Boche received the Research Award "Technische Kommunikation" from the Alcatel SEL Foundation in October 2003, the "Innovation Award" from the Vodafone Foundation in June 2006, and the Gottfried Wilhelm Leibniz Prize from the Deutsche Forschungsgemeinschaft (German Research Foundation) in 2008. He was a corecipient of the 2006 IEEE Signal Processing Society Best Paper Award and recipient of the 2007 IEEE Signal Processing Society Best Paper Award. He is a member of the IEEE Signal Processing Society SPCOM and SPTM Technical Committees. He was elected a member of the German Academy of Sciences (Leopoldina) in 2008.
Martin Schubert (M'05) received the diploma and doctoral degrees in electrical engineering from the Technische Universität Berlin, Germany, in 1998 and 2002, respectively.

In 1998 he joined the Heinrich-Hertz Institute for Telecommunications (HHI), Berlin, as a Research Assistant. Since 2003, he has been with the Fraunhofer German-Sino Lab for Mobile Communications (MCI), where he is working as a Senior Researcher. He is also a Lecturer with the Technical University of Berlin.

Dr. Schubert was a corecipient of the VDE Johann-Philipp-Reis Award in 2007. He coauthored the 2007 Best Paper Award of the IEEE Signal Processing Society. Since 2009, he has been an Associate Editor of the IEEE TRANSACTIONS OF Signal PROCESSING. 